\title{
Optimization of $\mathrm{CeBr}_{3}$ position-sensitive calorimeter module
}

\author{
Adrien Laviron ${ }^{\mathrm{a}, *}$, Valentin Gourlaouen ${ }^{\mathrm{a}}$, Clarisse Hamadache ${ }^{\mathrm{a}}$, Corentin \\ Hiver $^{\mathrm{a}}$, Jürgen Kiener ${ }^{\mathrm{a}}$, Jean Peyréa ${ }^{\mathrm{a}}$, Vincent Tatischeff ${ }^{\mathrm{a}}$ \\ ${ }^{a}$ Université Paris-Saclay, CNRS/IN2P3, IJCLab, 91405 Orsay, France
}

\begin{abstract}
For the next generation of $\mathrm{MeV}$ range gamma-ray telescopes, position sensitive calorimeters based on a monolithic scintillator coupled to a pixelated photodetector could be an important building block. In this paper, we present the optimization of the position reconstruction algorithms using machine learning, for a detector based on a $51 \times 51 \times 10 \mathrm{~mm}^{3} \mathrm{CeBr}_{3}$ crystal. For that purpose, we used an automated test bench and collimated radioactive sources to generate experimental data of known energy and position by irradiating the detector with gamma rays. We found in these data different gamma-ray interaction morphologies for which position reconstruction algorithms perform differently, and we developed an algorithm to automatically classify them. We also conducted an extensive optimization of the artificial neural networks that perform the 3D position reconstruction using the Keras Python library with Theano backend. We found that at $662 \mathrm{keV}, 90 \%$ of events have a morphology that facilitates position reconstruction. The optimised position reconstruction algorithms give for those events a rms error in the plane of the detector of $1.8 \mathrm{~mm}$ on each axis. The rms error in the depth of the crystal is found to be $2 \mathrm{~mm}$.
\end{abstract}

Keywords: Gamma--Ray Astronomy, Compton Imaging, Artificial

Neural Network, Scintillating Calorimeter, Cerium Bromide,

Position Reconstruction, Spatial Resolution

\footnotetext{
* Corresponding author

Email address: adrien.laviron@csnsm.in2p3.fr (Adrien Laviron)
} 


\section{Introduction}

Despite a large number of existing science topics, from nucleosynthesis and nuclear spectroscopy to multi-messenger astronomy and the extreme universe, gamma-ray astronomy in the $100 \mathrm{keV}$ to $10 \mathrm{MeV}$ energy range is understudied

5 due to the lack of sensitivity of current generation instruments. The best concept for next generation instruments is thought to be a space-borne Compton imager (with pair production imaging capabilities up to $100 \mathrm{MeV}$ ) [1. Constellations of nano satellites are also envisioned, because that concept would allow all-sky monitoring and provide a powerful insight in high-energy transient sources (see 10 e.g. [2] and references therein).

Compton imaging relies on the kinematics of Compton scattering which is the dominant process of interaction of gamma rays with matter in this energy range. Imaging gamma-rays using this principle was first proposed for astronomy in the $\mathrm{MeV}$ range [3] and nuclear medicine [4] in the 1970's. In an instrument as proposed in [1, the incoming photon undergoes a scattering in one or several layers of position-sensitive silicon strip detector before being absorbed in a position-sensitive calorimeter based on inorganic scintillators. The measurement of both positions and energy deposits provides information on the photon's source direction [5].

The localization accuracy and detection sensitivity achievable with this technique are also of interest for the localization of gamma-ray emitting radioactive material on Earth. Thus our results are also relevant for the ComptonCAM project [6], which aims at the development of an ultra sensitive, wide-field gamma camera for localization and characterization of low-intensity radioactive waste.

This study follows [7] and [8] on the development of a position-sensitive calorimeter module based on Cerium Bromide inorganic scintillator. In this paper we first introduce the experimental setup used to perform this study. We then present the characterization of the datasets, before explaining the methods used to reconstruct the position of interaction and discuss the results. 


\section{Experimental setup and measurements}

\subsection{Cerium bromide calorimeter module}

The calorimeter module used in this study is based on a square $51 \times 51 \times$ $10 \mathrm{~mm}^{3}$ Cerium Bromide $\left(\mathrm{CeBr}_{3}\right)$ inorganic crystal (manufactured by SCIONIX 35 company) coupled to a multi-anode photomultiplier tube (MAPMT). We then have with this setup a pixelated gamma-ray detector. Six calorimeter modules of this type have been characterized.

Because of the hygroscopic nature of the scintillating crystal used, it is embedded in an air-tight aluminium casing. One side of the crystal is coupled to a $1.5 \mathbf{~ m m}$ thick quartz optical window, itself coupled to the MAPMT using optical grease. The other sides of the crystal are wrapped in an optically diffusive layer of Teflon. The MAPMT used is a square, two-inches, $8 \times 8$ anodes Hamamatsu H12700A. Its photocathode side is $\mathbf{4 8 . 5} \mathbf{~ m m}$. It is read by a self-triggered electronic system ROSMAP (developped by IDEAS company), using four 16-channels ASICs and an FPGA to output the data in an Ethernet compatible format. Figure 1 shows a photography of the three components of the module.

In order to use this calorimeter module for Compton imaging, we need both a position and an energy measurement of each gamma-ray interacting in the crystal. We define an event as one or several interactions of a single gamma ray that deposits enough energy to trigger the read-out electronics.

For each event, the MAPMT measures the scintillation light distribution that enables the reconstruction of the gamma-ray's position of first interaction. The integral of the scintillation light distribution provides the energy deposit.

For the interaction position measurements, the key idea of this setup is to analyze the shape of the scintillation light distribution on the 64 pixels (64 charge values collected over the 64 anodes). Figure 2 shows an example of recorded event. From the shape of the light distribution we expect to determine the 3D coordinates (x,y (surface), $\mathrm{z}$ (depth)) of the gamma-ray first interaction

${ }_{60}$ in the crystal. Position reconstruction is based on machine learning algorithms, 


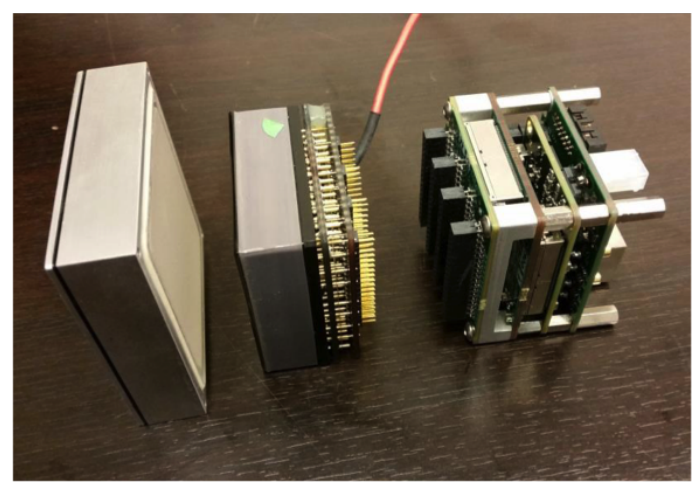

Figure 1: Photography (exploded view) of the calorimeter module. From left to right, we can see the scintillating crystal, the MAPMT and the read-out electronics

and will be discussed extensively in this paper.

\subsection{Test bench}

To assess the position reconstruction ability of the calorimeter module, we developed a test bench constituted of a radioactive source and a calorimeter module. The radioactive source is collimated to generate a gamma-ray beam directed towards this module.

For this study we used a combination of two $7.6 \mathrm{MBq}{ }^{137} \mathrm{Cs}$ sources emitting $662 \mathrm{keV}$ gamma rays, and a $424 \mathrm{kBq}{ }^{241} \mathrm{Am}$ source emitting $59.5 \mathrm{keV}$ gamma rays. In addition, we used two uncollimated sources, one $2.4 \mathrm{MBq}{ }^{133} \mathrm{Ba}$ source 70 emitting $81 \mathrm{keV}$ and $356 \mathrm{keV}$ gamma-rays, and one $37 \mathrm{kBq}{ }^{207} \mathrm{Bi}$ source emitting $570 \mathrm{keV}$ and $1064 \mathrm{keV}$ gamma-rays. The ${ }^{137} \mathrm{Cs}$ source is collimated using a $10 \mathrm{~cm}$ outer diameter, $10 \mathrm{~cm}$ long tungsten collimator for which the inner diameter can be changed. A good compromise between localization precision and detected source activity has been found at $1.3 \mathrm{~mm}$ inner diameter. The ${ }^{241} \mathrm{Am}$ source 75 is collimated using a $10 \mathrm{~cm}$ outer diameter, $1 \mathrm{~mm}$ inner diameter, $1 \mathrm{~cm}$ long copper collimator. The localization error associated with these collimators width contribute marginally to the calculated position reconstruction errors. It will be included in all results presented hereafter.

Background radioactivity measurements can be made by removing the source 

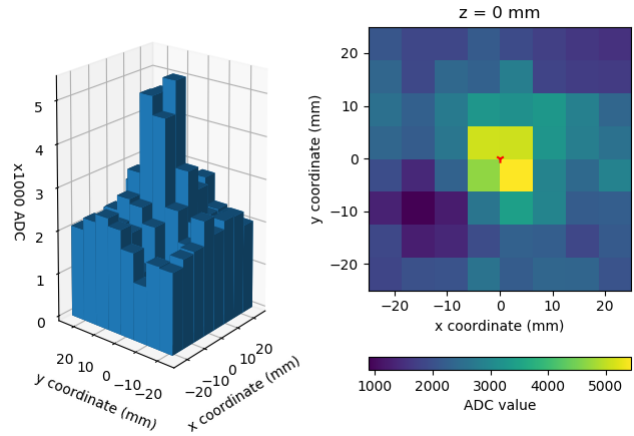

Figure 2: Example of an event recorded by the calorimeter module. Both parts of the figure shows the same data, one in parallel perspective and the other in colorscale. One can read on the $z$ axis or the color scale the quantity of light collected by each pixel of coordinates $(x, y)$.

or by using a full tungsten cylinder instead of the collimator. In order to reduce the background activity, the test bench is shielded by $4 \mathrm{~cm}$ thick lead bricks below it as well as on its four sides. A $5 \mathrm{~mm}$ copper plate can be added on the top of the calorimeter module to further reduce background at low energy.

The calorimeter module can be moved automatically to any position in a plan orthogonal to the beam direction by two worm gears driven by stepper motors controlled by an Arduino board. The Arduino is controlled through a serial port emulation by a LabVIEW program that also record the data from the calorimeter module. The module can also be rotated by $90^{\circ}$ for the beam to irradiate either its front or its side. Figure 3 shows both irradiation modes.

90 Figure 4 is a photography of the test bench in side irradiation configuration.

These properties of the setup aims at providing events of known coordinates and of known energy to perform the calibration of the calorimeter module. We call hereafter the coordinates of the beam the "mechanical positions". The coordinates system used in this study sets the origin in the center of the crystal.

95 The $x y$ plane spans the front of the detector, and the $z$ axis represents the depth of interaction (see Fig. 3). These coordinates are defined in the frame of the scanning bench by measuring the count rate in $0.5 \mathrm{~mm}$ steps to find the edges of the crystal. 

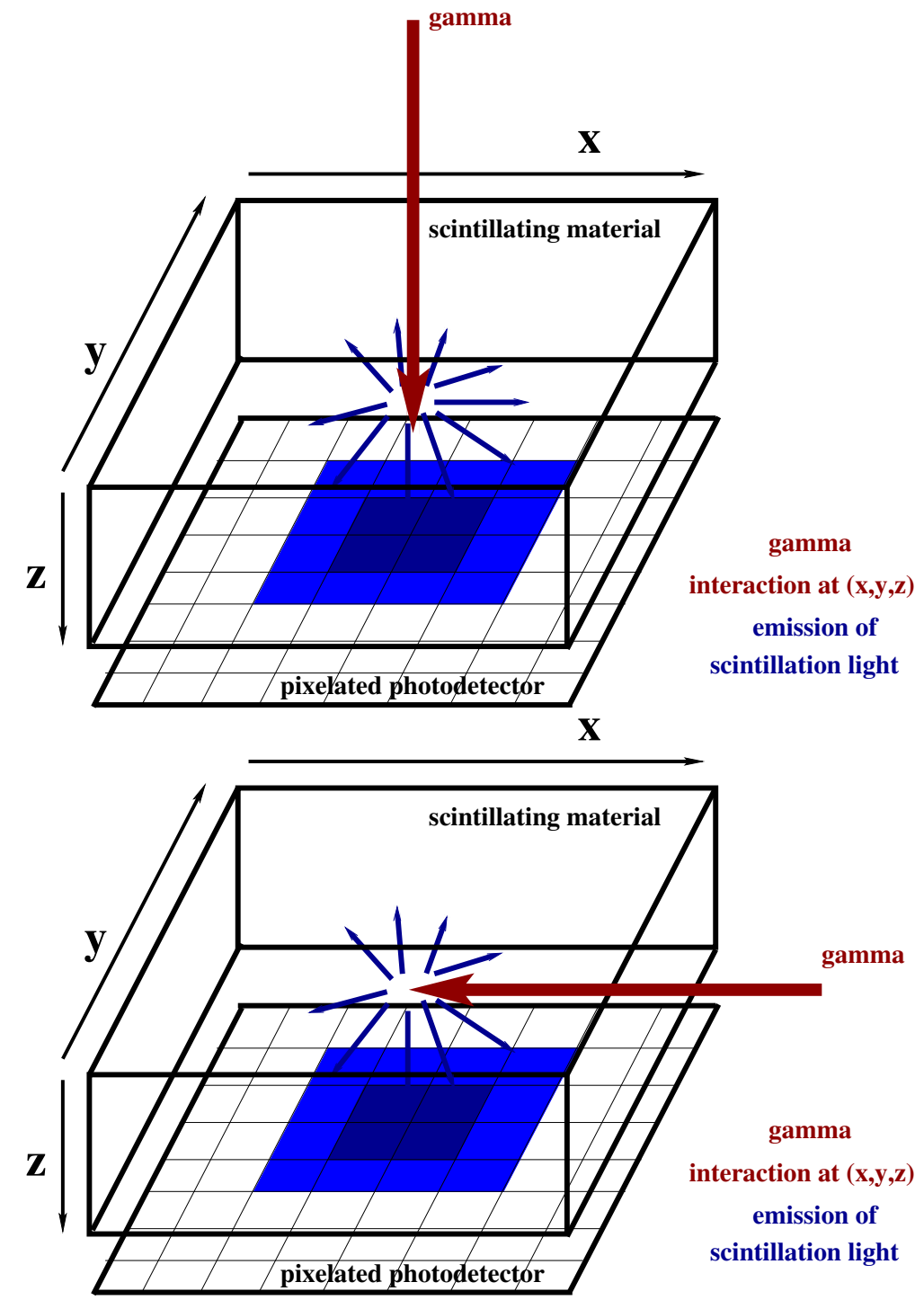

Figure 3: Scheme of the calorimeter module. The gamma-ray interacts in the $\mathrm{CeBr}_{3}$ that emits scintillation light. This light is detected by a pixelated photodetector optically coupled to the scintillating crystal. The shape of the scintillation light distribution provides information on the 3D position of the gamma-ray interaction. Top: front irradiation, $z$ in unknown. Bottom: side irradiation, $x$ in unknown 


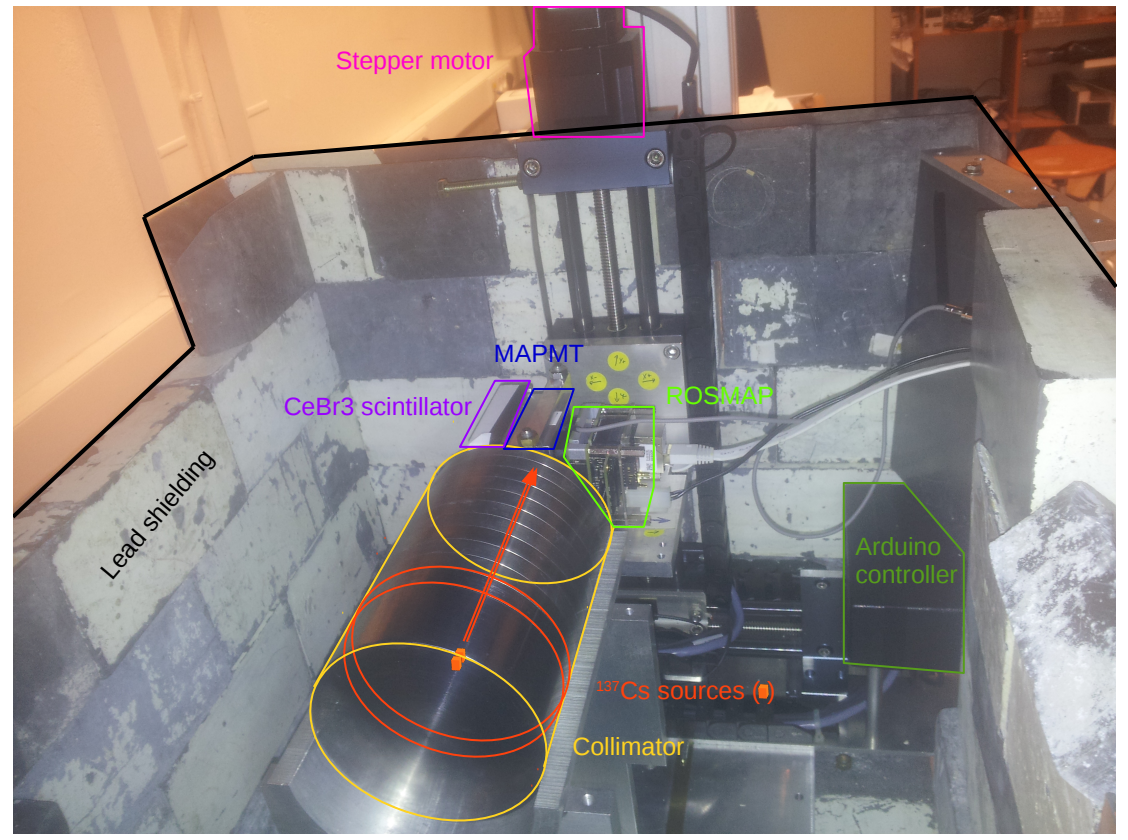

Figure 4: Photography of the automated test bench and collimated ${ }^{137} \mathrm{Cs}$ source. Source is framed in orange, collimator in yellow, $\mathrm{CeBr}_{3}$ scintillator, MAPMT and ROSMAP electronics respectively in purple, blue and light green. The case of the arduino board is framed in dark green, and the $y$ stepper motor in pink. The lead shieding is made of white and grey bricks and framed in black. 


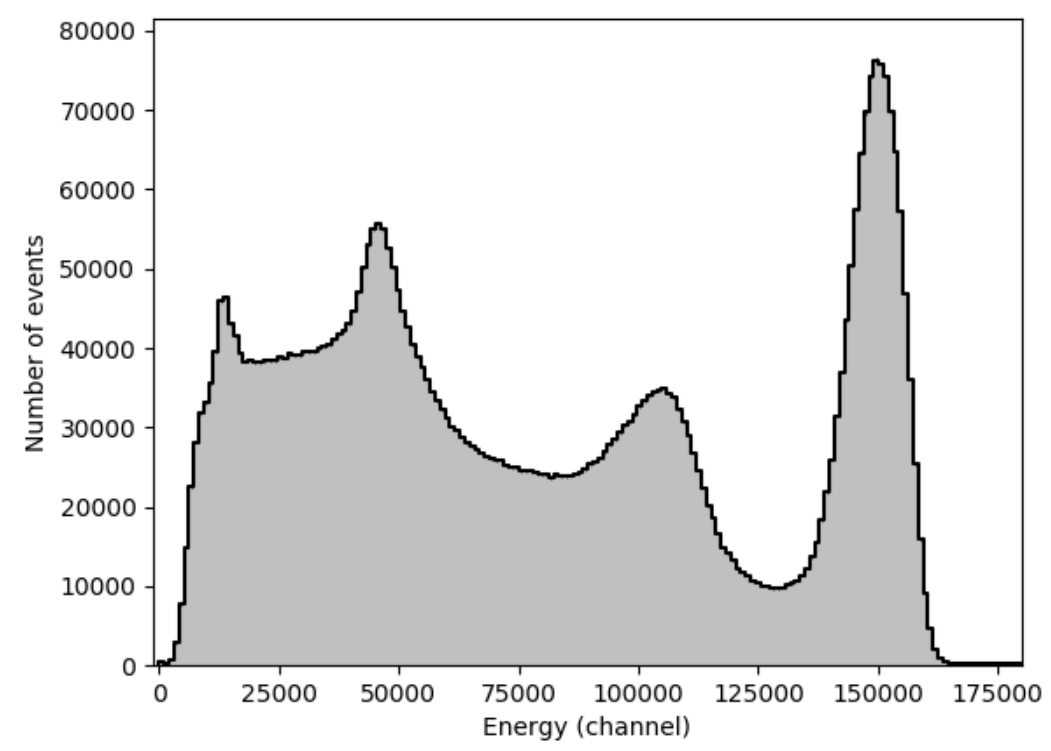

Figure 5: Spectrum of a ${ }^{137} \mathrm{Cs}$ source $(662 \mathrm{keV})$

\section{Data characterization}

\subsection{Spectra and energy selection}

Figure 5 shows a spectrum of a non collimated ${ }^{137} \mathrm{Cs}$ source aquired with this calorimeter module, obtained by simply summing the ADC values read by each anode. On the spectrum we can see the full-energy peak near channel 150,000 corresponding to $662 \mathrm{keV}$, the Compton front below channel 120, 000 and the backscattering peak near channel 45,000. Since we are designing a calorimeter, we will mostly be interested in the full-energy events for reference. However, simply selecting events in a given energy window is not optimal for two reasons

- The maximum of full-energy peak can be noticeably shifted from a mechanical position to another, as shown in Figure 6

- A broader than necessary energy selection allows more background radioactivity events in the datasets 


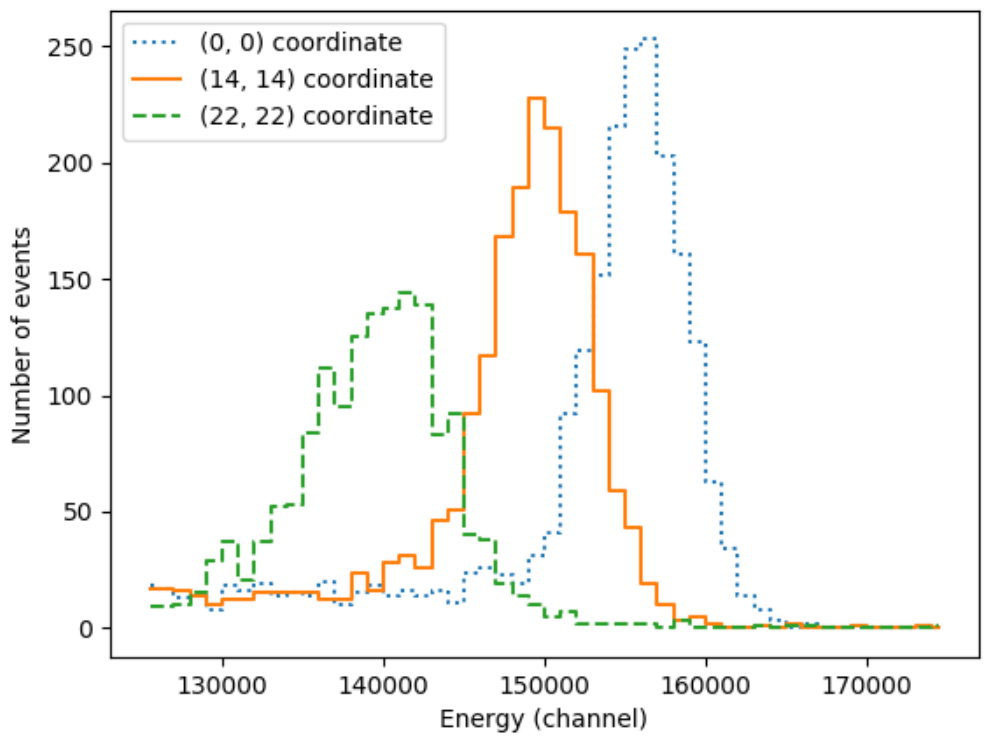

Figure 6: Spectra of a collimated ${ }^{137} \mathrm{Cs}$ source at different mechanical positions, $(0,0)$ being the center of the crystal, $(14,14)$ an intermediary position and $(22,22)$ a position close to a corner. 
For these reasons, a finer energy selection, depending on the mechanical position, has been performed on the datasets. For each mechanical position, a gaussian function is fitted to the full-energy peak and events of energy inside a

\subsection{Corrections to improve spectral resolution}

The energy resolution obtained by simply summing the charge of the 64 anodes is about $8.3 \%$ at $662 \mathrm{keV}$. This unexpected high value for a $\mathrm{CeBr}_{3}$ scintillator is due to the position-dependent full energy peak spectral shift shown in Figure 6 .

That effect has already been noticed at a smaller scale on smaller scintillators 9 . It can be explained by the loss of scintillation photons at the interface between the crystal and the reflector. Because of absorption and diffusion (studied in detail in $[8$ ), the collected charge is higher near the center than close to PMT can also explain part of these shifts, since those gains can vary by a factor of 2 . However, this effect is found to be less important than the former, as shown on Figure 7, on which the spectral shifts are represented before and after the gain correction. It shows that the but not on its non-uniformity. This correction will not be applied in the following, unless it is explicitly stated.

Let's now consider a reference mechanical position, the center of the crystal for example, so that the spectral shift is defined as a shift with respect to this position's spectrum. The shift can now be expressed as the ratio of the full-energy peak's maximum at the reference position to the full-energy peak's maximum 

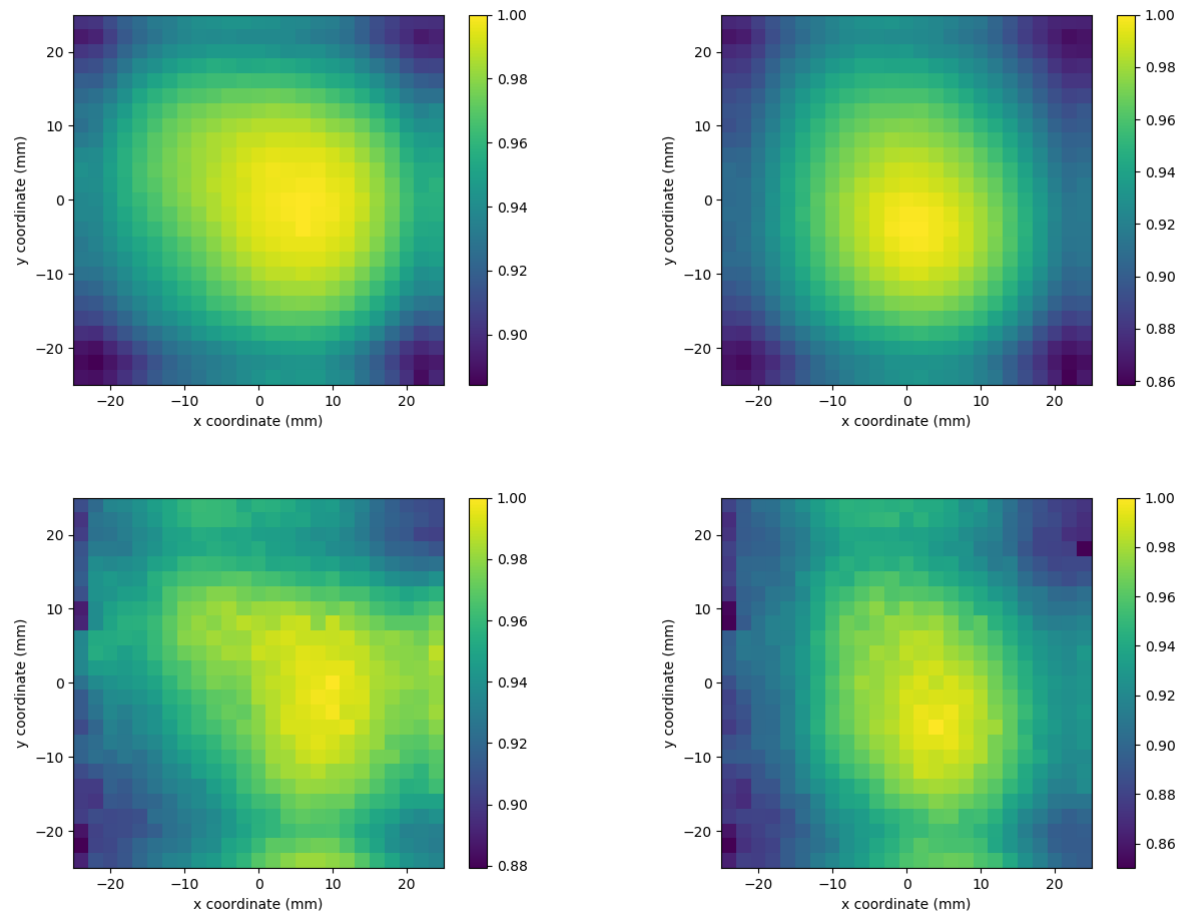

Figure 7: Relative detector spectral response through its surface, as given by the position of the center of a gaussian fitted to a spectral line, for $25 \times 25$ mechanical positions with a $2 \mathrm{~mm}$ pitch. Top: measured for the $662 \mathrm{keV}$ line of ${ }^{137} \mathrm{Cs}$. Bottom: measured for the $59.5 \mathrm{keV}$ line of ${ }^{241} \mathrm{Am}$. Left: before correction of the variable gain of the 64 anodes. Right: after correction. (colour online)

at another position. This quantification allows for an energy correction for each event by a multiplicative factor, as long as the coordinates of the event are reconstructed.

It should be noted that this method takes into account part of the anode gain differences, even though the correction is not applied. Indeed, the scintillation light distribution can be modelled as a continuum and a peak (see Figure 2). The anode gain correction has no effect on the contribution of the continuum to the measured energy, and the peak position is largely determined by the position of inter- 

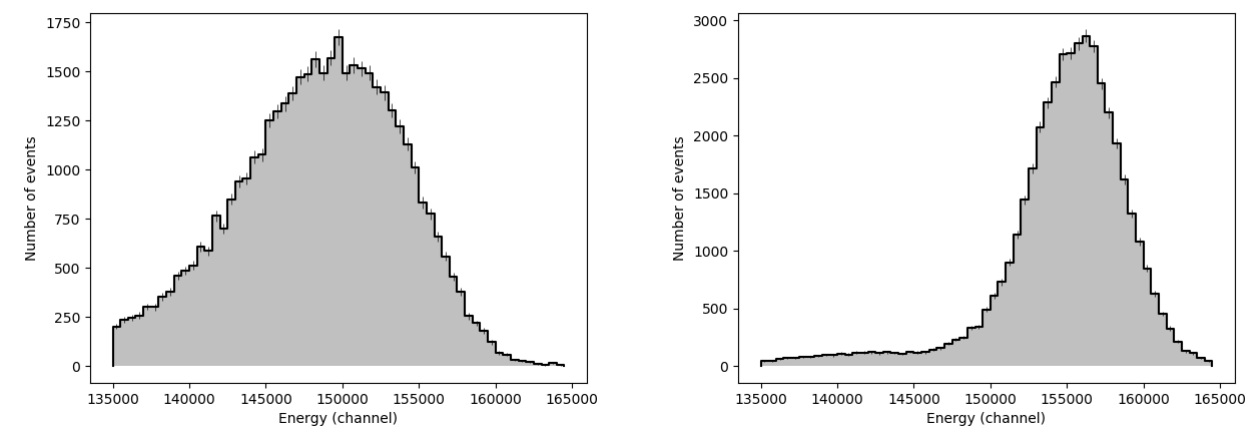

Figure 8: Left: simple spectrum, with spectral resolution of $8.3 \%$ at $662 \mathrm{keV}$. Right: corrected spectrum, with spectral resolution of $4.7 \%$ at $662 \mathrm{keV}$

action. Therefore, a correction based on the position of interaction corrects part of the anode non-uniformity.

An example of this method's results is shown on Figure 8, where the spectral resolution improves from $8.3 \%$ to $4.7 \%$ at $662 \mathrm{keV}$. The same correction with the same coefficient gives good results at other energies, see e. g. Figure 9 for the $570 \mathrm{keV}$ and $1064 \mathrm{keV}$ lines of ${ }^{207} \mathrm{Bi}$ for which we had respectively $8.3 \%$ and $7.8 \%$ FWHM spectral resolution and attain respectively $4.8 \%$ and $4.4 \%$. In this last case, this method is probably limited by our ability to precisely reconstruct the position of interaction at high energies (see Section 4.2).

\subsection{Morphology of events}

Data analysis by visual examination of the events' scintillation light distributions showed a variety of morphologies. Those morphologies can be classified in three main categories as illustrated in Fig. 10 . The impact of these morphologies on position reconstruction will be discussed in Section 4.2.5.

The first category presents a single, localised energy deposit. The second category presents two, identifiable and localised energy deposits. The third category presents an energy deposit that is wide and continuous. It should be noted that this classification discretizes a continuous physical phenomenon and therefore that some events are borderline cases. 

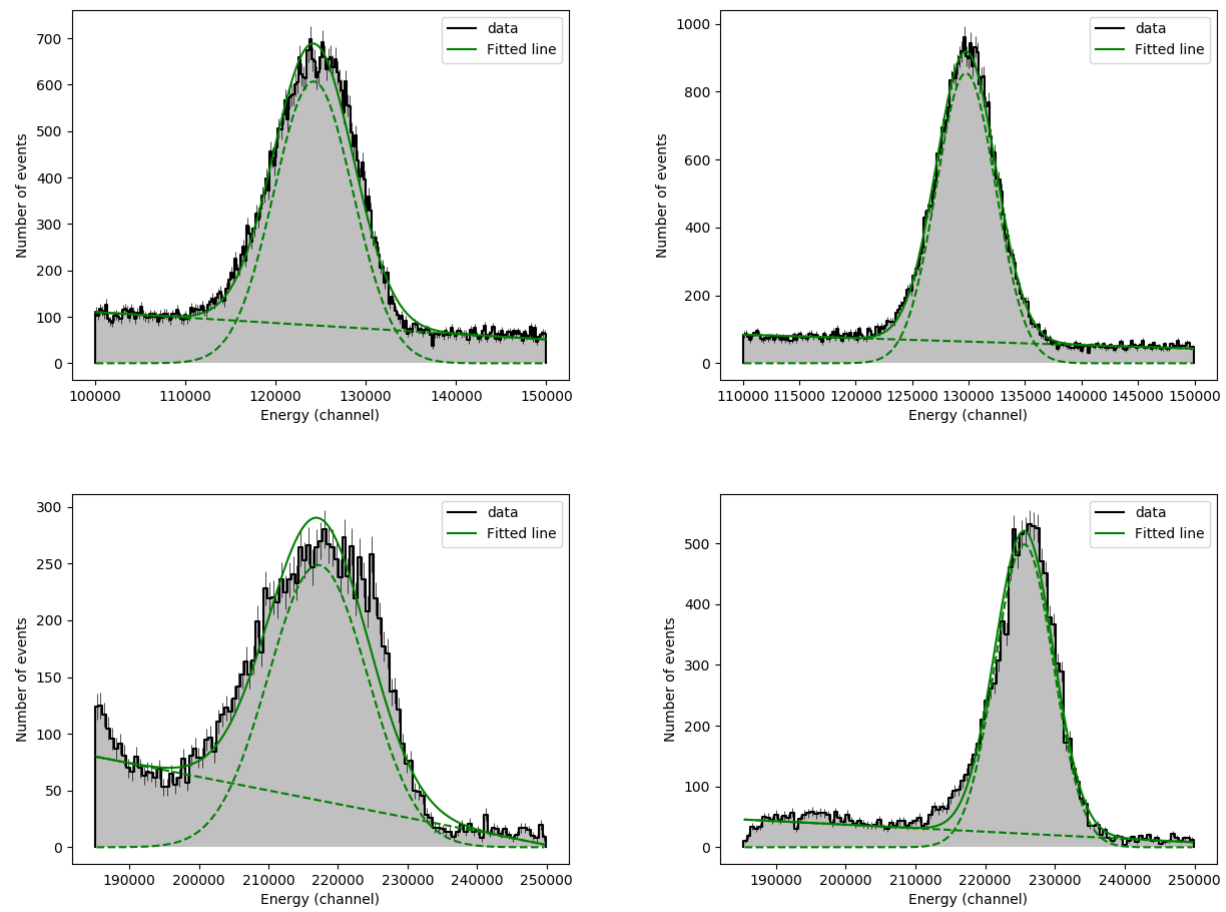

Figure 9: Left: raw spectrum of the $570 \mathrm{keV}$ (top) and $1064 \mathrm{keV}$ (bottom) lines of a ${ }^{207} \mathrm{Bi}$ radioactive source. Right: corrected spectrum, showing the two lines with respectively $4.8 \%$ and $4.4 \%$ FWHM spectral resolution. 

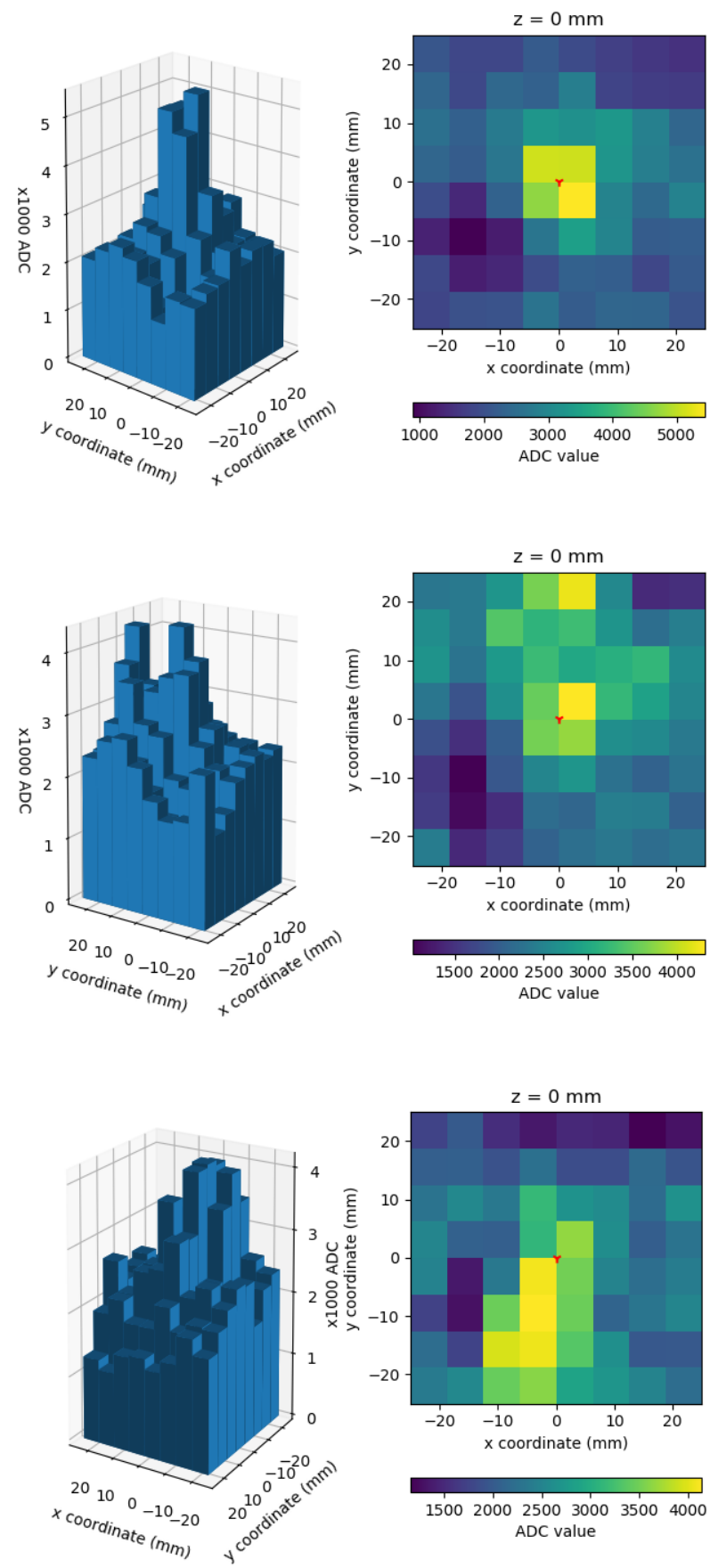

Figure 10: Three events with different morphologies. Data are represented twice, once in parallel perspective (left) and once in colorscale (right). The red mark in the colorscale representation indicates the mechanical position. Top: single, localised energy deposit. Center: two energy deposits. Bottom: wide, continuous energy deposit. 
developed. Its core idea is to assess if a significant amount of light is spread or clustered. For that purpose we choose, amongst all pixels, those that have received the most light. The number of pixels considered is a parameter of this algorithm. This selection is made after applying to the pixels the correction of the gains of the anodes. Then we define a shape (typically a square) that we consider acceptably clustered. This is also a parameter of the algorithm. Finally, we check that the considered pixels can fit in the shape. This check relies on a two-dimensional convolution of the scintillation light distribution, masked with chosen pixels, by the shape. If such a position exists, the event is selected.

The event selection procedure uses this algorithm two times sequentially. First, we select the events for which the 4 considered pixels are found in a $2 \times 2$ pixels square. The events that are not selected by this first selection go through a second round, during which are selected those for which the 5 considered pixels can be found in a $3 \times 3$ pixels square.

This two-stage selection is necessary because the second round will select events that have been rejected by the first one but have a shifted or truncated scintillation light distribution, especially on the borders of the crystal. But it should be noted that events selected during the first round could have been rejected by the second. Indeed, choosing too many pixels includes in the analysis pixels from the light continuum that have the highest values because of photon noise, discarding events that have an acceptable morphology.

This procedure selects between $89 \%$ and $94 \%$ of events (depending on the calorimeter module characterized) in datasets recorded with a ${ }^{137} \mathrm{Cs}$ source and selected by the fine energy selection around $662 \mathrm{keV}$ described in Section 3.1 and between $85 \%$ and $93 \%$ of events selected the same way around the $356 \mathrm{keV}$ line of a ${ }^{133} \mathrm{Ba}$ source. This result shows that most of events at these energies have a localised energy deposit in the crystal.

This procedure is meaningless for events below a certain energy because not 200

enough scintillation light is produced. At low energy, as shown on Figure 11 with 

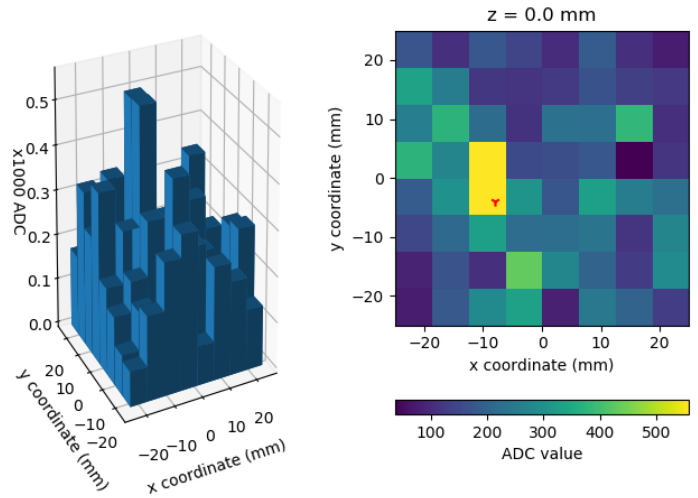

Figure 11: Event recorded from a ${ }^{241} \mathrm{Am}$ radioactive source.

an event of energy $59.5 \mathrm{keV}$, most events will have scintillation light distribution compatible with a single, localised energy deposit, because gamma-rays interact in $\mathrm{CeBr}_{3}$ mostly via photoelectric absorption.

\section{Position reconstruction}

\subsection{Artificial neural networks}

An artificial neural network (ANN) is a network of simple algorithms that aims at mimicking the operation of a biological neuron. Those algorithms execute a weighted sum of several inputs, and apply to this number an $\mathbb{R} \rightarrow \mathbb{R}$ function called activation function. We use ANNs to reconstruct the position of the first interaction of a gamma ray from the measured scintillation light distribution. The general idea of this position reconstruction method has been proposed in [10] for positron emission tomography scanners.

The machine learning used in this study is supervised learning. It means that ANNs are first trained to output the correct answer, using input data with known output, by minimizing an error function. For our case, the input data are the 64 channels of our MAPMT, the output is the real position of interaction of the gamma ray in the crystal. The mechanical positions approximate the 
known outputs. We choose for our error function a mean square error. Once the training is completed, reconstructing unknown events consists in executing the neural network program with the parameters calculated during the training process. The weights of the neurons are free parameters adjusted during the training of the ANN.

ANNs used in this work are multi-layer perceptrons: neurons are organised into one or several layers, that are executed sequentially. Such non-cyclic neural networks have been described in 1943 in [11. The number of layers and their size (number of neurons) are the parameters of the ANN chosen by the programmer. Layers outside of the input and output ones are called "hidden layers". The activation function is also such a parameter, as well as the algorithm used for training. These parameters define the neural network's architecture.

During training, the neurons weights are adjusted by iterations, called epochs [12], to minimize the error from the reconstructed gamma-ray interaction coordinates to the mechanical position. After a certain number of epochs, this error decreases without improving the general ANN's performance. This phenomenon is called overlearning. In this case the neural network tends to reconstruct better the events from the training dataset, but looses performance for any other event. To prevent overlearning, the error function is also calculated on a second dataset, not used for the training and called validation dataset. The training is stopped whenever this second error stops decreasing. All this work has been done using the Python Keras framework [13] with Theano [14] backend.

In this part we will present the effect of various event selection algorithms and neural network architectures on the performance of position reconstruction, first in 2D in the $x y$ plane (see Fig. 3), and then in the depth of the crystal along the $z$ axis. 


\subsubsection{Datasets}

The aim of this part is to investigate the performances attainable with ANN based $x y$ position reconstruction. For that purpose, datasets were acquired with the automated test bench. A first dataset, used for training, contains

\subsubsection{Performance indicators}

This study also investigates the effects of the ANN's architecture on the position reconstruction performance. For the $x y$ reconstruction, the performance of an ANN is measured by way of its mean uncertainty $\bar{\sigma}_{\mathrm{XY}}$, defined as the quadratic average on all mechanical positions $p$ of the $\sigma_{\mathrm{XY}, p}$,

$$
\bar{\sigma}_{\mathrm{XY}}=\sqrt{\frac{1}{N} \sum_{p} N_{p} \sigma_{\mathrm{XY}, p}^{2}}
$$

where $N_{p}$ is the number of events at mechanical position $p$. The quantity $\boldsymbol{\sigma}_{\mathbf{X Y}, \boldsymbol{p}}$ is defined as the square root of the mean squared distance from the 
reconstructed $x$ and $y$ coordinates (in the frame defined on Fig. 3 ) to the known ones,

$$
\sigma_{\mathrm{XY}, p}=\sqrt{\frac{1}{N_{p}} \sum_{i=1}^{N_{p}}\left[\left(x_{p}-x_{i}^{\mathrm{rec}}\right)^{2}+\left(y_{p}-y_{i}^{\mathrm{rec}}\right)^{2}\right]},
$$

where $N_{p}$ is the number of events at the mechanical position $p, x_{p}$ (respectively $\left.y_{p}\right)$ is the known $x$ (resp. $y$ ) coordinate of the mechanical position $p$ and $x_{i}^{\text {rec }}$ (resp. $y_{i}^{\text {rec }}$ ) is the $x$ (resp. $y$ ) coordinate of event $i$ reconstructed using the ANN.

In order to quantify any systematic offset between the known position and the reconstructed position, an offset error $\sigma_{\mathrm{XY}, \boldsymbol{p}}^{\mathbf{o}}$ has been defined as

$$
\sigma_{\mathrm{XY}, p}^{\mathrm{O}}=\sqrt{\left(x_{p}-\bar{x}\right)^{2}+\left(y_{p}-\bar{y}\right)^{2}}
$$

${ }_{275}$ where $\bar{x}(\operatorname{resp} . \bar{y})$ is the mean of the values of the $x_{i}^{\text {rec }}\left(\right.$ resp. $y_{i}^{\text {rec }}$ ) of events at this mechanical position (see Section 2.2.

The $\sigma_{\mathrm{XY}, p}$ can be expressed as the quadratic sum of $\sigma_{\mathrm{XY}, p}^{\mathrm{o}}$ and a point spread error

$$
\sigma_{\mathrm{XY}, p}^{\mathrm{s}}=\sqrt{\frac{1}{N_{p}} \sum_{i=1}^{N_{p}}\left[\left(x_{i}^{\mathrm{rec}}-\bar{x}\right)^{2}+\left(y_{i}^{\mathrm{rec}}-\bar{y}\right)^{2}\right]},
$$

\subsubsection{Exploration of $A N N^{\prime}$ 's architecture parameters space}

The analysis has been performed for all combinations of the following architecture parameters:

- 0 to 4 hidden layers

- 6 to 35 neurons per hidden layer

- 10 different activation functions [13]: sigmoid, hard sigmoid, tanh, elu, relu, softplus, softmax, softsign, exponential, and linear

- 3 training algorithms: adam, nadam and adamax 

the value of $\bar{\sigma}_{\mathrm{XY}}$, in $\mathrm{mm}$, attained after training, calculated on a set of $N=$ 14450 events evenly distributed on the $x y$ plane of the detector.

Such output has been studied also for the mean point spread error $\bar{\sigma}_{\mathrm{XY}}^{\mathrm{s}}$ (see Eq. 4) and the mean offset error $\bar{\sigma}_{\mathrm{XY}}^{\mathrm{O}}$ (see Eq. 3) as shown on Figure 13 . This analysis shows that the offset error is systematically lower than the point spread error. Some architectures, using elu or softplus activation functions and more than 16 neurons per hidden layer, perform better than the others on this test. Moreover, some architectures such as those using the softsign display a point spread error better than most, at the cost of a larger offset error. Architectures using more neurons do not seem to overlearn because of the validation dataset monitoring. However, they require more calculations, wich may be of critical importance depending on the application.

\subsubsection{Energy dependency}

This study has been performed in two different energy ranges, one around the full-energy peak of a ${ }^{137} \mathrm{Cs}$ source at $662 \mathrm{keV}$ and one around the $59.5 \mathrm{keV}$ line of a ${ }^{241} \mathrm{Am}$ source. In this second energy range, the same fine energy selection has been applied. No morphology selection was made as discussed in Section 3.3

To assess the energy dependency of neural networks, the same analysis has been conducted with 50 events per mechanical position, recorded at energies near $662 \mathrm{keV}$ and near $59.5 \mathrm{keV}$. This study showed that using two neural networks, each trained with dataset from a single $59.5 \mathrm{keV}$ or $662 \mathrm{keV}$ energy window, does not perform significantly better than using a single neural network trained with a dataset composed with both energy windows.

Finally, we conclude that, for the $x y$ position reconstruction and the set of architectures studied here, there is no significant improvement over 2 layers and $\approx 19$ neuron per hidden layer (see Fig. 12). The best training algorithm for our purpose appear to be adamax, and both elu and softplus activation functions give good results. In the following, we use one ANN for all energies and the elu 


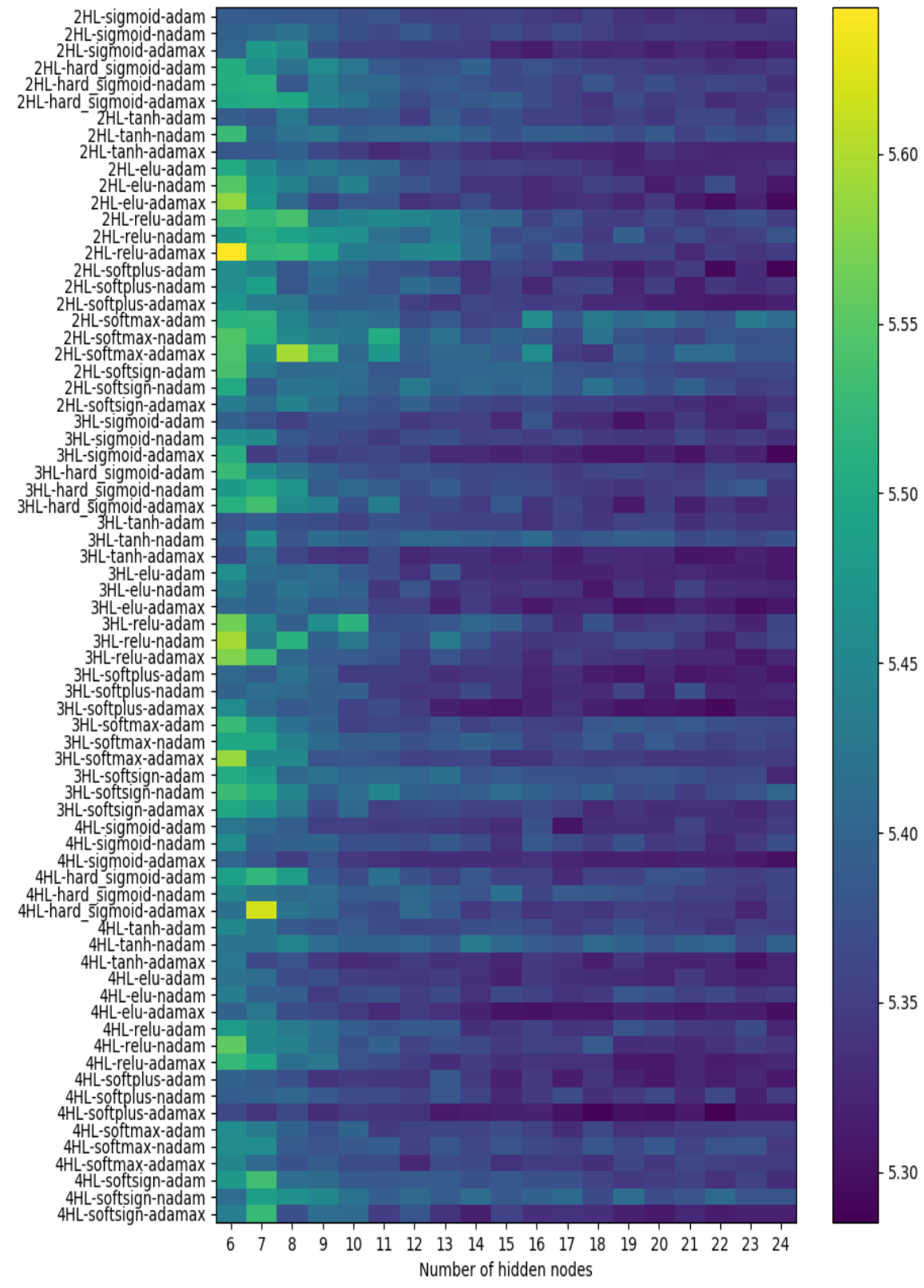

Figure 12: Output example of a systematic ANN's architecture parameter space exploration. On the $x$ axis is represented the number of neurons per layer. On the $y$ axis are indicated the number of hidden layers (HL), the activation function and the training algorithm used. Colorscale represents the performance of the ANN $\overline{\boldsymbol{\sigma}}_{\mathbf{X Y}}$ in $\mathbf{m m}$ (lower is better, see Eq. 1]. (colour online) 

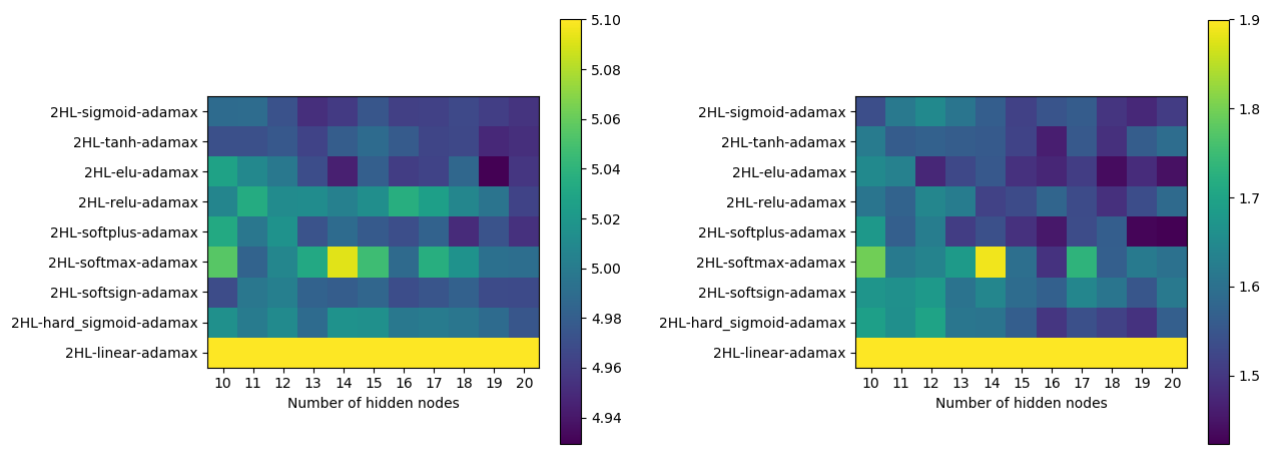

Figure 13: Ouput of an exploration of some architectures for neural networks with 2 hidden layers and adamax training algorithm. Left: colorscale represents $\bar{\sigma}_{\mathrm{XY}}^{\mathrm{s}}$ Right: colorscale represents $\bar{\sigma}_{\mathrm{XY}}^{\mathrm{o}}($ colour online $)$

activation function by default.

\subsubsection{Position resolution}

An example of reconstructed dataset is shown on Fig. 14. That dataset contains 200 events per mechanical position, that passed the fine energy cut at $662 \mathrm{keV}$ described in Section 3.1 and the morphology cut described in Section 3.3 .

We see that most gamma rays are reconstructed within a short distance of their associated mechanical position, and with a good consistency throughout the detector. The events at mechanical positions near the border of the scintillating material are harder to reconstruct because their scintillation light distribution is truncated or misshapen. We still obtain $\bar{\sigma}_{\mathrm{XY}}=5.0 \mathrm{~mm}$ on that dataset.

Some events are reconstructed very far from their associated mechanical position. They are interpreted as background radioactivity. It should be noted that all calculations of the ANN performance $\bar{\sigma}_{\mathrm{XY}}$ (as well as $\bar{\sigma}_{\mathrm{XY}}^{\mathrm{s}}$ and $\bar{\sigma}_{\mathrm{XY}}^{\mathrm{o}}$ ) using Eq. 1 (respectively Eq. 4 and Eq. 3 ) include some events from the background radioactivity. To assess its contribution to the position resolution, we recorded a dataset with the ${ }^{137} \mathrm{Cs}$ source at a single mechanical position 


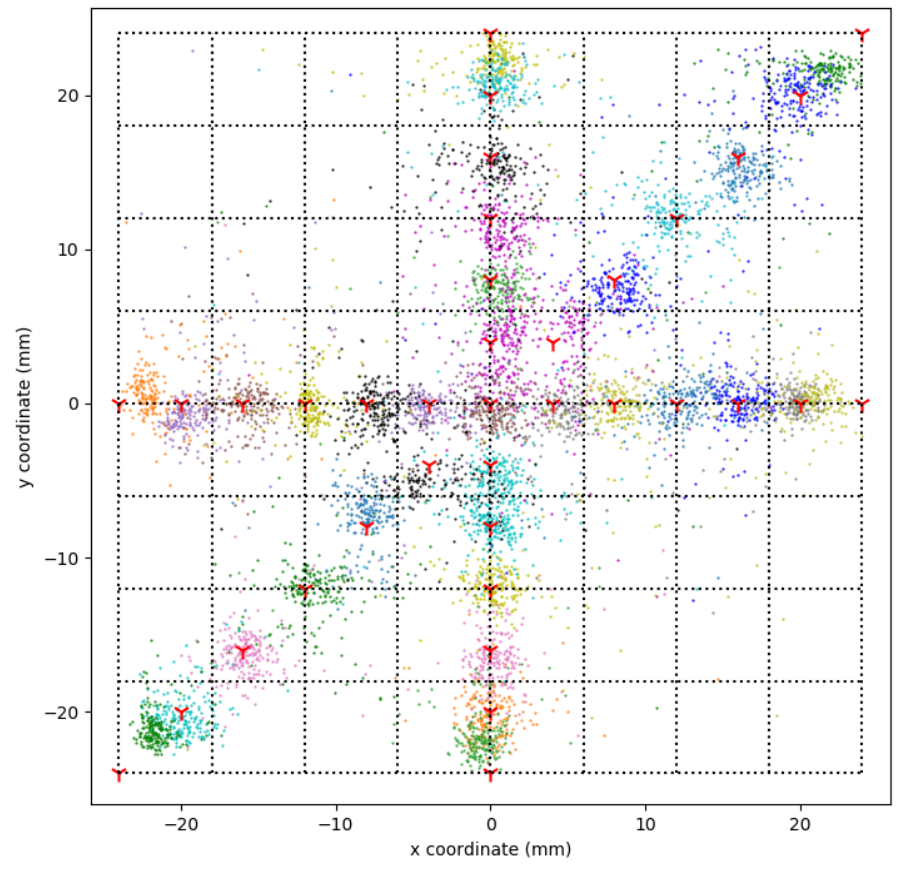

Figure 14: Example of a dataset's position reconstruction by our ANN. Mechanical positions change by steps of $4 \mathrm{~mm}$ along $x$ and $y$ axes, and 200 events of energy $662 \mathrm{keV}$ were selected for each mechanical position. Events were selected by energy and morphology. Mechanical positions are indicated by the red markers, and gamma-rays reconstructed coordinates shown as colored dots. A single (but not unique) color is associated to a mechanical position. Dotted black lines show the borders of the pixels. 

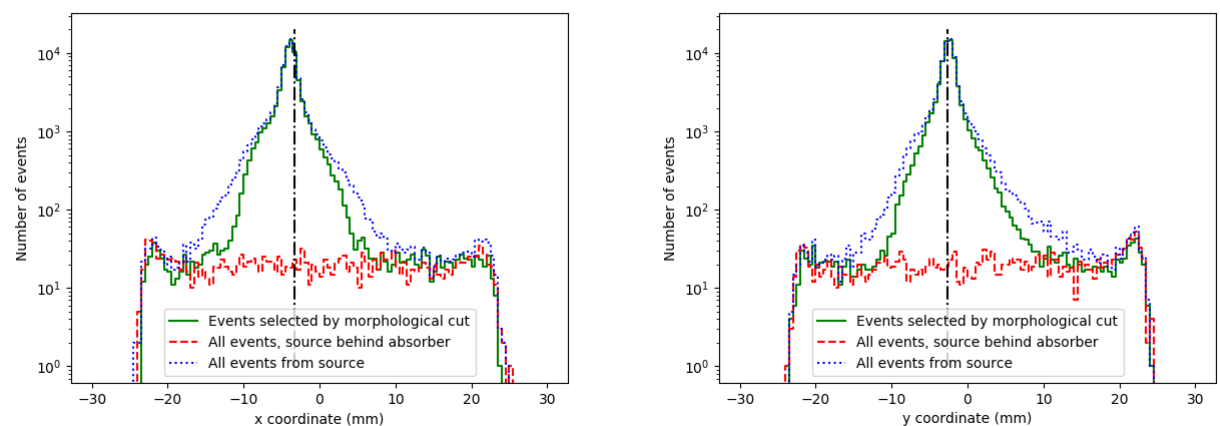

Figure 15: 1D histograms of reconstructed $\boldsymbol{x}$ and $\boldsymbol{y}$ coordinates for a single mechanical position $x=-3.3 \mathrm{~mm}, y=-2.6 \mathrm{~mm}$. Left: projection along the $x$ axis. Right: projection along the $y$ axis. The blue dotted lines represent the reconstructed coordinates of events in the dataset acquired with the source. The green solid lines represent the reconstructed coordinates of events from the same dataset that have been selected for their morphology. The red dashed lines represent the reconstructed coordinates of events from the background radioactivity dataset (source behind absorber). The black dashed-dotted vertical lines represent visually the known coordinates.

$(x=-3.3 \mathrm{~mm}, y=-2.6 \mathrm{~mm})$ with a $1 \mathrm{~mm}$ inner diameter collimator. The background radioactivity has been estimated by placing a thick tungsten absorber between the source and the detector, as described in Section 2.2, and recording data for the same duration (two hours). Events were selected using a large energy window between $\approx 580 \mathrm{keV}$ and $\approx 700 \mathrm{keV}$. A histogram of the reconstructed gamma-ray positions of interaction can be seen on Figure 15 , The blue lines represent the reconstructed coordinates of all events, the green lines the reconstructed coordinates of morphologically selected events, and the red lines the background radioactivity. The blue and green lines meet the red one at large distance from the position of interaction, which validates the interpretation that the events that are reconstructed far enough from the known position of interaction are background radioactivity.

When the background radioactivity is subtracted bin to bin to the data in the histogram, a new estimation of the performance of the ANN $\sigma_{\mathbf{X Y}, p}^{\prime}$ can be 


\begin{tabular}{|l|c|c|c|c|c|c|}
\hline $\begin{array}{l}\text { Morphology } \\
\text { selection }\end{array}$ & $\sigma_{\mathrm{X}, p}$ & $\sigma_{\mathrm{Y}, p}$ & $\sigma_{\mathrm{XY}, p}$ & $\sigma_{\mathrm{X}, p}^{\prime}$ & $\sigma_{\mathrm{Y}, p}^{\prime}$ & $\sigma_{\mathrm{XY}, p}^{\prime}$ \\
\hline Not applied & $3.6 \mathrm{~mm}$ & $3.5 \mathrm{~mm}$ & $5.0 \mathrm{~mm}$ & $2.9 \mathrm{~mm}$ & $2.6 \mathrm{~mm}$ & $3.9 \mathrm{~mm}$ \\
\hline Applied & $3.0 \mathrm{~mm}$ & $2.9 \mathrm{~mm}$ & $4.2 \mathrm{~mm}$ & $1.9 \mathrm{~mm}$ & $1.7 \mathrm{~mm}$ & $2.6 \mathrm{~mm}$ \\
\hline
\end{tabular}

Table 1: Summary of the position resolution measured at the position $x=-3.3 \mathrm{~mm}, y=$ $-2.6 \mathrm{~mm}$

calculated for a single mechanical position $\boldsymbol{p}$ as

$$
\sigma_{\mathrm{XY}, p}^{\prime}=\sqrt{\frac{1}{N} \sum_{b i n=1}^{n} N_{b i n} \times\left[\left(x^{\mathrm{known}}-x_{b i n}^{\mathrm{rec}}\right)^{2}+\left(y^{\mathrm{known}}-y_{b i n}^{\mathrm{rec}}\right)^{2}\right]}
$$

where $N$ is the number of events in the dataset after subtraction, $n$ the number of bins in the histogram, $N_{b i n}$ the number of events in a given bin, $x^{\text {known }}$ and $y^{\text {known }}$ the mechanical position of the dataset $(x=-3.3 \mathrm{~mm}, y=-2.6 \mathrm{~mm})$, $x_{b i n}^{\mathrm{rec}}$ and $y_{b i n}^{\mathrm{rec}}$ the coordinate of the center of the bin bin.

It gives, at this position $\boldsymbol{p}, \sigma_{\mathrm{XY}, p}^{\prime}=3.9 \mathrm{~mm}$ for all events and $\sigma_{\mathrm{XY}, p}^{\prime}=$ ${ }_{50} \quad 2.6 \mathrm{~mm}$ for morphologically selected events. The results are presented in Table 1 for each axis. They point at an average localization error of $1.8 \mathrm{~mm}$.

These $\sigma_{\mathrm{XY}, p}$ and $\sigma_{\mathrm{XY}, p}^{\prime}$ should not be interpreted as the standard deviation of a gaussian distribution. Figure 16 shows the histograms of the difference 35 from the reconstructed to the known coordinates (as in Figure 15 but in linear scale), in the dataset used to evaluate $\bar{\sigma}_{\mathrm{XY}}$, together with a gaussian fit that provides a poor description of the data, and especially of the distribution tails. The standard deviations of the fitted gaussians are $\approx 1.55 \mathrm{~mm}$ at $662 \mathrm{keV}$ and $\approx 1.7 \mathrm{~mm}$ at $59.5 \mathrm{keV}$ for both the $x$ and $y$ axes.

As shown on Figure 17, the variations of the position resolution $\sigma_{\mathrm{XY}, p}$ (for which background radioactivity has not been subtracted) is dominated by the loss of performance in the $\approx 5 \mathrm{~mm}$ near the borders of the crystal. 

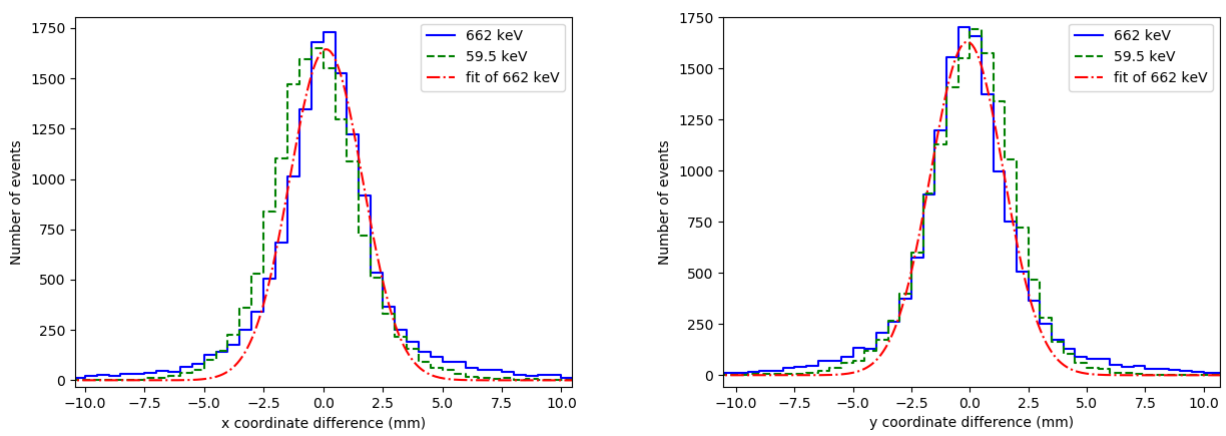

Figure 16: 1D histograms of the difference between the reconstructed and known coordinates. Left: $x$ coordinates. Right: $y$ coordinates. The blue solid lines represent the $662 \mathrm{keV}$ dataset, for which the selection on event morphology has been applied. The green dashed lines represent the $59.5 \mathrm{keV}$ dataset. The red dotted-dashed line show a gaussian fit of the distribution at $662 \mathrm{keV}$.

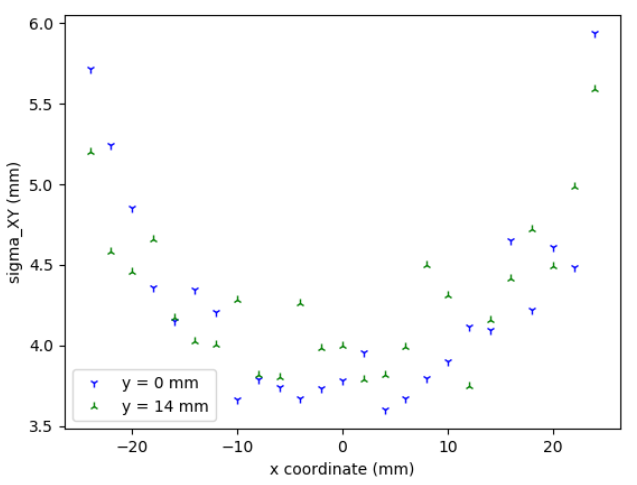

Figure 17: Position resolution estimator $\sigma_{\mathrm{XY}, p}$ uniformity through the detector $x$ axis, calculated using morphologically selected events at $662 \mathrm{keV}$ with one data point every two millimeters. Blue: at $y=0 \mathrm{~mm}$. Green: at $y=14 \mathrm{~mm}$ 


\subsection{Depth of interaction reconstruction}

interaction along the $z$ axis (see Fig. 3). Since the depth of interaction is unknown when irradiating the front of the crystal, acquiring data that allow neural networks training requires new procedures, which will be presented in Section 4.3.1. New performance indicators will be defined (Section 4.3.2 to be conducted, presented in Section 4.3.3. Finally, the results of artificial neural networks will be compared against methods based on the known absorption length of radiation in the crystal in Section 4.3.4.

\subsubsection{Data acquisition and selection}

375 tion, the detector is irradiated from the side (see Fig. 3 in Section 2.2.

We use $662 \mathrm{keV}$ photons from the ${ }^{137} \mathrm{Cs}$ source to be able to explore all the crystal, since gamma rays below $\approx 300 \mathrm{keV}$ do not penetrate enough in the crystal. We then obtain data of different energies by selecting events in the plateau, events mostly result from a single interaction of a gamma ray in the crystal. Therefore, selection on event morphology is not needed. Since the gamma rays have an initial energy of $662 \mathrm{keV}$, absorption is no longer a problem. This method allows to probe all $x, y$ and $z$ positions in the crystal with scintillation light distribution of a lower energy photon depositing all its energy by the scintillation light distribution of a Comptonised electron of the same energy. The difference should be negligeable, given that the mean free path of the generated low energy electrons in the $\mathrm{CeBr}_{3}$ is $<0.1 \mathrm{~mm}$ and that the photodetector pixels are $6 \mathrm{~mm}$ wide.

Three energy ranges were defined in addition to the full-energy peak, around $300 \mathrm{keV}$ between the Compton front and the backscattering peak, around $130 \mathrm{keV}$ below the backscattering peak, and near $50 \mathrm{keV}$. In those energy ranges, no fine 
energy selection (as defined in Section 3.1) could be applied because there is no peak to fit. No selection on event morphology was performed because the selection on event energy already selects single interaction events.

For each of these energy ranges, the dataset used for training spans the whole $y z$ plane of the detector with a $1 \mathrm{~mm}$ step. The gamma-ray absorption in $\mathrm{CeBr}_{3}$ at these energies is such that events may interact at any $x$ coordinate for each $y z$ known coordinates. After the energy selection (and fine energy selection in the energy range near $662 \mathrm{keV}), 200$ events are selected for each mechanical position, out of which $30 \%$ will be used for validation.

\subsubsection{Performance indicators}

To assess the performance of different methods on the position reconstruction along the $z$ axis, a new performance indicator $\bar{\sigma}_{\mathrm{Z}}$ has been defined as the quadratic mean on all mechanical position $p$ of all $\sigma_{\mathrm{Z}, p}$, themselves defined as the one-dimensionnal square root of the mean squared distance,

$$
\sigma_{\mathrm{Z}, p}=\sqrt{\frac{1}{N_{p}} \sum_{i=1}^{N_{p}}\left(z_{p}-z_{i}^{\mathrm{rec}}\right)^{2}},
$$

where $z_{p}$ is the known $z$ coordinate of the mechanical position $p$ and $z_{i}^{\text {rec }}$ is the $z$ coordinate reconstructed using a given method.

Just as $\bar{\sigma}_{\mathrm{XY}}^{\mathrm{S}}$ and $\bar{\sigma}_{\mathrm{XY}}^{\mathrm{o}}$ were defined in Section 4.2 .2 (see Eq. 4 and Eq. 3 ), we define a mean point spread error $\bar{\sigma}_{\mathrm{Z}}^{\mathrm{s}}$ and a mean offset error $\bar{\sigma}_{\mathrm{Z}}^{\mathrm{O}}$ as the quadratic mean on all mechanical positions $p$ of the

$$
\begin{gathered}
\sigma_{\mathrm{Z}, p}^{\mathrm{s}}=\sqrt{\frac{1}{N_{p}} \sum_{i=1}^{N_{p}}\left[\left(z_{i}^{\mathrm{rec}}-\bar{z}\right)^{2}\right]} \\
\sigma_{\mathrm{Z}, p}^{\mathrm{o}}=\left|z_{p}-\bar{z}\right|
\end{gathered}
$$

where $\bar{z}$ is the mean of the $z_{i}^{\text {rec }}$ values for all events recorded at given mechanical position $p$ (see Section 2.2. 
Even though the $y$ coordinate is known for each of these events, it will not be reconstructed here. For the neural network, first investigations on the reconstruction of the depth of interaction showed that asking for the reconstruction of both $y$ and $z$ coordinates led to poor results in $z$ reconstruction, because $y$ is both easier to reconstruct and spans a larger range of values, so the training algorithm tends to prioritize that coordinate. Therefore, the $y$ coordinate will not be used in this part.

\subsubsection{Exploration of ANN's architecture parameters space}

Given the weak dependency of the performance of neural networks on the total number of neurons, shown by preliminary tests, this part of the analysis focused on the activation function and training algorithm optimization. The analysis has been performed for the following set of architecture parameters:

- 4 hidden layers

- 80 neurons per hidden layers

- 10 activation functions [13: sigmoid, hard sigmoid, tanh, elu, relu, softplus, softmax, softsign, exponential, and linear

- 3 training algorithms: adam, nadam and adamax

For our purpose, the training algorithm that performed the best was adamax. The most performing activation functions were elu and relu. We will use elu in the following. The energies at which depth of interaction is best reconstructed are around $662 \mathrm{keV}$ and $300 \mathrm{keV}$, with $\bar{\sigma}_{\mathrm{Z}} \approx 2 \mathrm{~mm}$. In the $130 \mathrm{keV}$ energy range, ANNs are significantly worse with $\bar{\sigma}_{\mathrm{Z}} \approx 2.4 \mathrm{~mm}$, and in the $50 \mathrm{keV}$ energy domain they perform even worse with $\bar{\sigma}_{\mathrm{Z}} \approx 2.6 \mathrm{~mm}$. This could be explained by the lower number of scintillation photons generated by the interaction, which gives a noisier measured scintillation light distribution, source $\mathrm{X}$-rays from the cesium $\mathrm{K} \alpha$ line at $31 \mathrm{keV}$, disturbing the ANN training. 


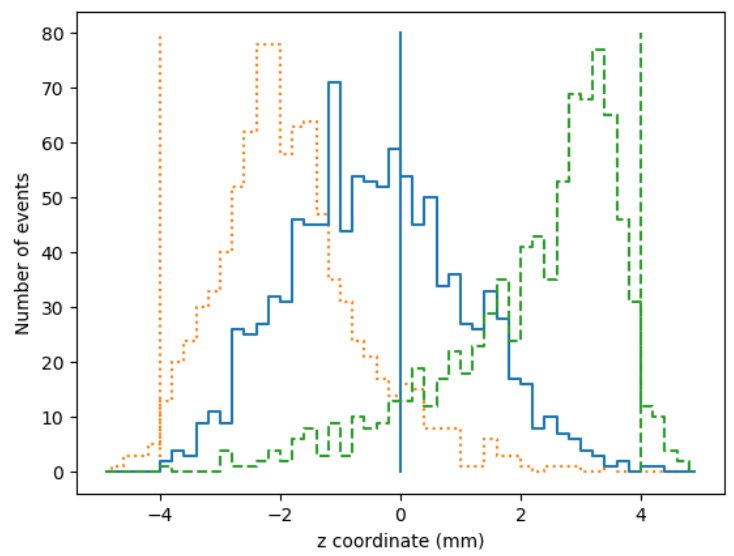

Figure 18: Histogram of reconstructed depths of interaction at $662 \mathrm{keV}$. Yellow dotted: at $y=10 \mathrm{~mm}, z=-4 \mathrm{~mm}$. Blue solid: at $y=0 \mathrm{~mm}, z=0 \mathrm{~mm}$. Green dashed: at $y=$ $10 \mathrm{~mm}, z=4 \mathrm{~mm}$. Vertical lines show visually the known depth of interaction

As shown on Figure 18, some $z$ coordinates near the edges of the crystal are hard to reconstruct, namely $z \in[-5,-4]$ and $z \in[4,5]$. Figure 19 show the values of $\sigma_{\mathrm{Z}, p}$ and $\sigma_{\mathrm{Z}, p}^{\mathrm{s}}$, represented in colorscale, for all $y$ and $z$ coordinates. It confirms that the error is dominated by the poor reconstruction abilities near the edges of the crystal.

Finally, as for the 2D $(x, y)$ position reconstruction, training ANNs with all events from the $662 \mathrm{keV}, 300 \mathrm{keV}$ and $130 \mathrm{keV}$ energy domains led to $\bar{\sigma}_{\mathrm{Z}}$ results similar to those obtained with specific ANNs trained with events from each energy domain.

Figure 20 shows the histograms of the difference between the reconstructed and known $z$ coordinate. As energy decreases, the reconstruction of the depth of interaction becomes less precise and more asymetric towards over-estimated depths.

The combination of the two optimised ANN allow 3D position reconstruction, as shown on Figure 21 

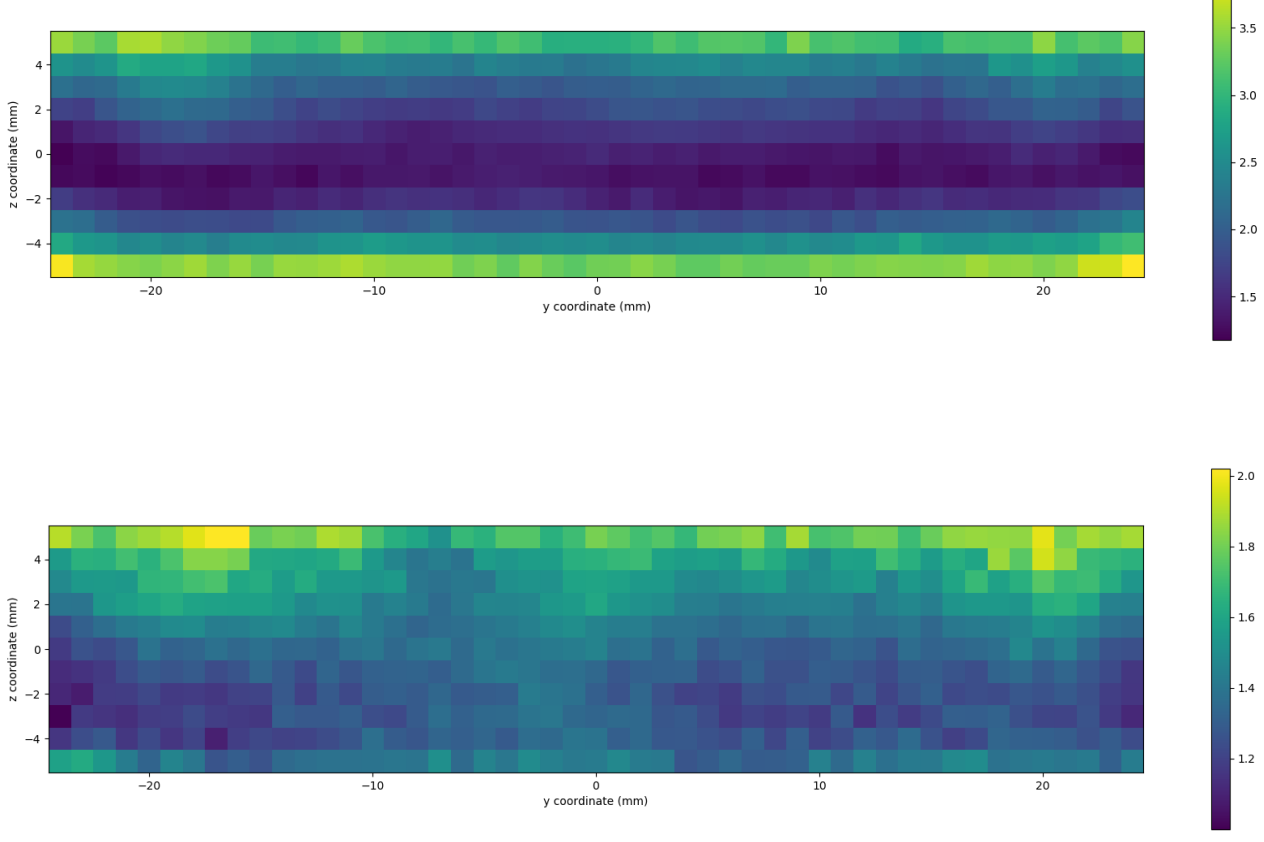

Figure 19: $\sigma_{\mathrm{Z}, p}$ (top) and $\sigma_{\mathrm{Z}, p}^{\mathrm{s}}$ (bottom) represented in colorscale in $\mathrm{mm}$ throughout the $y z$ plane. (colour online) 


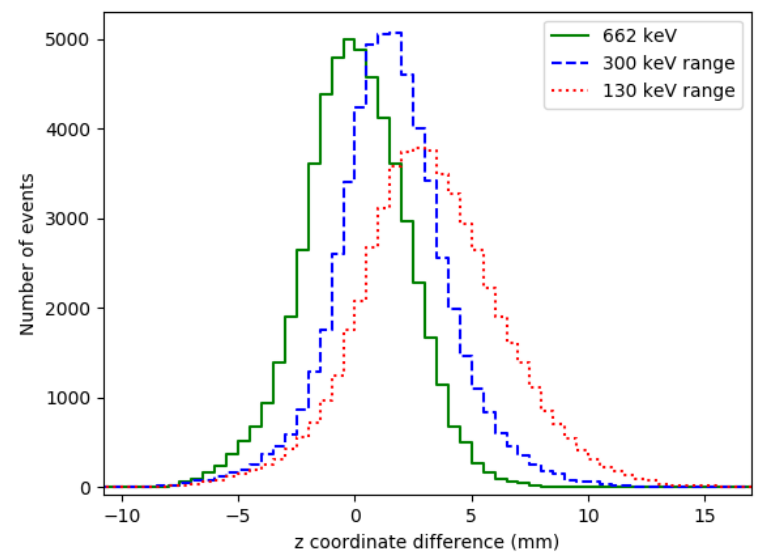

Figure 20: 1D histogram of the distance between the reconstructed and known depth of interaction. The green solid line represents the results at $662 \mathrm{keV}$, the blue dashed line in the $300 \mathrm{keV}$ range and the red dotted line in the $130 \mathrm{keV}$ range

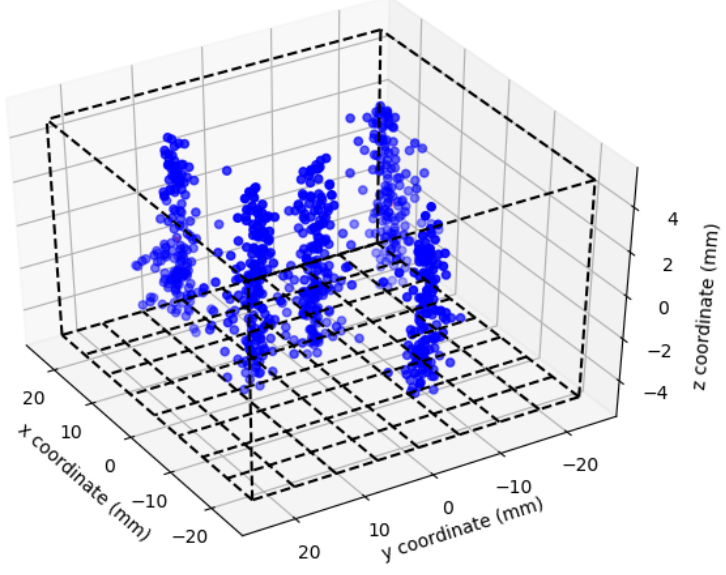

Figure 21: 3D position reconstruction using two optimised ANN for $5 x y$ mechanical positions. 


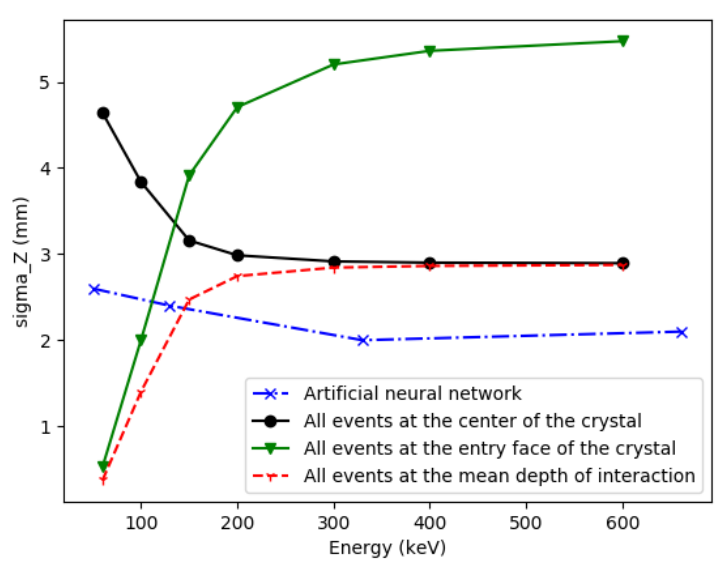

Figure 22: $\bar{\sigma}_{\mathrm{Z}}$ (in $\mathrm{mm}$ ) versus the energy of incoming gamma-ray for various methods. ANN's results are shown in blue. The black curve represents the error achieved when all events are reconstructed at $z=0 \mathrm{~mm}$. The green curve represents the error achieved when all events are reconstructed at $z=-5 \mathrm{~mm}$. The red curve represents the error achieved when all events of a given energy are reconstructed at their mean depth of interaction.

\subsubsection{Depth of interaction reconstruction at low energies}

At energies lower than $100 \mathrm{keV}$, the attenuation length of gamma rays in $\mathrm{CeBr}_{3}$ is lower than $2 \mathrm{~mm}$, therefore using a neural network that provides an uncertainty on position reconstruction larger than $2 \mathrm{~mm}$ is subopti-

mal. Instead, two main methods could be used: the simplest one consists in assigning to every event, independently of its scintillation light distribution, a fixed $z$ coordinate. Below $\approx 100 \mathrm{keV}$, an appropriate coordinate is $z=-5 \mathrm{~mm}$, that is the coordinate of the entry face of the crystal. A more complex method is to assign to every event a depth of interaction that depends only on its measured energy, and equal to the mean of several simulated depth of interaction in the crystal. This simulation approach allows to easily account for the finite size of the crystal. Depths are calculated using the NIST XCOM photon cross-section database data 15 .

Figure 22 represents the $\bar{\sigma}_{\mathrm{Z}}$ error (see Eq. 6) achieved with different depth 
of interaction reconstruction methods. Apart from the ANN results, these data are simulated with a Monte-Carlo method. The green and black curves show the results of assigning to every event the coordinate $z=-5 \mathrm{~mm}$ and $z=0 \mathrm{~mm}$ (center of the crystal), respectively. The choice of $z=0 \mathrm{~mm}$ is better at high energies because then the absorption is not dominant. The red line represents the error achieved by the "mean depth of interaction" method. At high energies, since the mean goes to $z=0 \mathrm{~mm}$, we find back the results of this method. At low energies, it is always better than the $z=-5 \mathrm{~mm}$ approach because it uses a better model of the reality. We note that these methods rely on the assumption that a low energy deposit is due to a gamma ray coming from the front of the detector and being absorbed, which may or may not be the case depending on the situation. Finally, neural networks show a better performance than any other method above $\approx 150 \mathrm{keV}$.

\section{Results and discussion}

We studied the performance of a gamma-ray detection module made of a $\mathrm{CeBr}_{3}$ monolithic scintillating crystal coupled to a 64 -channels MAPMT. We developed algorithms to optimize the spectral resolution and 3D position resolution.

We obtain a spectral resolution ehancement down to $4.7 \%$ FWHM thanks to a position-dependent energy correction, as presented in Section 3.2 . This correction does not require a strict calibration of the ADC to charge relation nor a correction for the different gains of the 64 anodes, and it is fairly lightweight from a computational perspective once the gamma-ray position of interaction is reconstructed.

495

The selection on event morphology allows a better position reconstruction as shown on Figure 15. It also leads to a better understanding of the data. A $662 \mathrm{keV}$ gamma ray in $\mathrm{CeBr}_{3}$ will in most cases interact via Compton scattering. However, at this energy, photons are mostly scattered in the forward direction. This may explain why a majority of events shows a localised 


\begin{tabular}{|l|c|c|c|c|c|c|}
\hline Module number & 0 & 1 & 2 & 3 & 4 & 5 \\
\hline $\bar{\sigma}_{\mathrm{XY}}$ at $59.5 \mathrm{keV}$ & $4.6 \mathrm{~mm}$ & $4.5 \mathrm{~mm}$ & $4.3 \mathrm{~mm}$ & $5.1 \mathrm{~mm}$ & $4.6 \mathrm{~mm}$ & $4.9 \mathrm{~mm}$ \\
$\bar{\sigma}_{\mathrm{XY}}$ at $662 \mathrm{keV}$ & $5.1 \mathrm{~mm}$ & $4.0 \mathrm{~mm}$ & $4.7 \mathrm{~mm}$ & $4.2 \mathrm{~mm}$ & $4.1 \mathrm{~mm}$ & $4.1 \mathrm{~mm}$ \\
\hline $\begin{array}{l}\text { Fraction of events } \\
\text { selected at } 662 \mathrm{keV}\end{array}$ & $85 \%$ & $90 \%$ & $89 \%$ & $93 \%$ & $93 \%$ & $94 \%$ \\
\hline $\begin{array}{l}\bar{\sigma}_{\mathrm{XY}} \text { at } 662 \mathrm{keV} \\
\text { after morphology } \\
\text { selection }\end{array}$ & $4.9 \mathrm{~mm}$ & $3.7 \mathrm{~mm}$ & $4.3 \mathrm{~mm}$ & $3.8 \mathrm{~mm}$ & $3.7 \mathrm{~mm}$ & $3.7 \mathrm{~mm}$ \\
\hline
\end{tabular}

Table 2: Summary of results obtained in $x y$ position reconstruction and morphology analysis for the six modules characterised.

energy deposit resulting of several, closely packed gamma-ray interactions. It can also explain the large continuous energy deposits. The third identified morphology, showing several distinct energy deposits, can then be interpreted as several Compton interactions. The fractions of selected events at $662 \mathrm{keV}$ presented here varies between $89 \%$ and $94 \%$, except for one module for which $85 \%$ of events were selected. At $356 \mathrm{keV}$, this fraction varies from $85 \%$ to $93 \%$, and is $81 \%$ for this one module (detailed results are presented in Table 2). The behavior of that module may be explained by a small anomaly in the optical coupling between the crystal housing and the PMT. The preferred forward scattering depends on the energy, so this variation of the fraction of selected events was expected. Future work could further investigate this variable. This event selection based on morphology allow a position resolution improvement from $\sigma_{\mathrm{XY}, p}^{\prime}=3.9 \mathrm{~mm}$ to $\sigma_{\mathrm{XY}, p}^{\prime}=2.6 \mathrm{~mm}$ at $662 \mathrm{keV}$. It may or may not be used in the context of a Compton space observatory, depending on the expected flux of the source.

Investigations on the data that were discarded for their morphology shows that neural networks probably cannot reconstruct them correctly. Indeed, when neural networks are trained using only these events, all tested architectures output $\bar{\sigma}_{\mathrm{XY}}>7 \mathrm{~mm}$. However, neural networks trained only with events 
having that unaccepted morphology and a maximum of the scintillation light distribution located near the mechanical position give great results, down to $\bar{\sigma}_{\mathrm{XY}}=4 \mathrm{~mm}$ (including background radioactivity events). This cannot be implemented in an actual Compton imager, since the real position of interaction is not known. But from that analysis, we can assume that neural networks reconstruct these events at the position of the highest energy deposit, which may or may not be the position of first interaction.

The position reconstruction in the $x y$ plane has been optimised down to an uncertainty of $1.8 \mathrm{~mm}$ for all tested energies. Following investigations showed that this method can be generalized to other calorimeter modules with the same geometry and electronics, even though the gain of individual PMT anodes may vary by a factor 2. Quantitative results for the six modules that have been characterized are reported in Table 2, We found the same ANN architecture to perform very well for all calorimeter modules: 2 hidden layers, 19 neurons per hidden layer using the elu activation function and trained using the adamax algorithm. However, that requires a new neural network training with data from that module.

Preliminary investigations showed that a single neural network could also be used with several calorimeter modules, if it has been trained with a combined set of data from each of those modules, with minimal performance loss, even though the individual anode gains of the PMT were not corrected in this study. At $662 \mathrm{keV}$, the neural network dedicated to the module 1 yielded $\bar{\sigma}_{\mathrm{XY}}=4.57 \mathrm{~mm}$, and the one dedicated to the module 2 , $\bar{\sigma}_{\mathrm{XY}}=4.50 \mathrm{~mm}$, while the neural network trained with data from both modules gave for each module, respectively, $\bar{\sigma}_{\mathrm{XY}}=4.56 \mathrm{~mm}$ and $\bar{\sigma}_{\mathrm{XY}}=4.52 \mathrm{~mm}$. For comparison, neural networks that were trained with a single module give consistently $\bar{\sigma}_{\mathrm{XY}}>6 \mathrm{~mm}$ on the data from other modules. Future work will investigate further this result, as it may have a significant impact on the dataflow design of future instruments. Future work could also investigate the ability of such software to reconstruct the position of interaction in thicker crystals, since those 

in a low count rate application such as a space telescope.

The results obtained in position and spectral resolution fulfill the requirements of the calorimeter of next generation space-borne Compton telescopes. Moreover, it has a position resolution noticeably finer than its pitch $(1.8 \mathrm{~mm}$ for $6 \mathrm{~mm}$ square detectors), reducing the number of channels to read at the cost of some computation. The effect of this tradeoff should be discussed for embedded applications such as space observatories and nano-satellites in which both electrical power and computer resources for data management are limited.

The characteristics of the module we developed make it also an interesting 560 detector for a portable gamma camera aimed at locating and characterising radioactive waste following the dismantling of nuclear facilities [6].

Depth of interaction reconstruction is less critical than $x y$ position reconstruction in the context of a Compton telescope, and especially since our calorimeter modules are not very thick $(10 \mathrm{~mm})$. However, its importance increases as the scattering detector and the calorimeter get closer. The neural network approach yielded good results at the cost of an increase in ANN complexity for the medium and high energy events $(>150 \mathrm{keV})$. The optimised $z$ ANN architecture uses 4 hidden layers and 80 neurons per hidden layer using the elu activation function, and is trained using the adamax training algorithm. At low energy, the simple method based on the interaction length of gamma rays in $\mathrm{CeBr}_{3}$ gives satisfactory results.

\section{Conclusions}

For the next generation of $\mathrm{MeV}$ range gamma-ray telescopes, position sensitive $\mathrm{CeBr}_{3}$ scintillating detectors could be an important building block. We studied six detectors using that technology and developed a position-dependent energy correction, an event selection procedure that recognises event morphologies and we optimised the position reconstruction algorithms. We attained an energy resolution of $4.7 \%$ FWHM at $662 \mathrm{keV}$ with the energy correction. 
The 3D position reconstruction of a gamma-ray interaction relies on two

artificial neural networks, one for the plane $x y$ of the detector and one for its depth $z$. The optimised $x y$ ANN architecture uses 2 hidden layers and 19 neurons per hidden layer using the elu activation function. The optimised $z$ ANN architecture uses 4 hidden layers and 80 neurons per hidden layer using the elu activation function. Both were trained using the adamax training algorithm. We found the optimised ANN architectures to perform equally on different calorimeter modules for a wide energy range (from $60 \mathrm{keV}$ to $662 \mathrm{keV}$ ). The $z$ position can be reconstructed with $\mathrm{a} \approx 2 \mathrm{~mm}$ error. The $x y$ position resolution depends on the morphology of events, but a $1.8 \mathrm{~mm}$ error has been attained for $\approx 90 \%$ of events.

These results make that calorimeter module technology a very appealing choice for the next generation of $\mathrm{MeV}$ range gamma-ray space borne observatories.

\section{Acknowledgements}

This work benefited from the Investments for the future program of the French Government and operated by the French National Radioactive Waste Management Agency (ANDRA).

\section{References}

[1] A. De Angelis, V. Tatischeff, M. Tavani, U. Oberlack, I. Grenier, L. Hanlon, R. Walter, A. Argan, P. von Ballmoos, A. Bulgarelli, I. Donnarumma, M. Hernanz, I. Kuvvetli, M. Pearce, A. Zdziarski, A. Aboudan, M. Ajello, G. Ambrosi, D. Bernard, E. Bernardini, V. Bonvicini, A. Brogna, M. Branchesi, C. Budtz-Jorgensen, A. Bykov, R. Campana, M. Cardillo, P. Coppi, D. De Martino, R. Diehl, M. Doro, V. Fioretti, S. Funk, G. Ghisellini, E. Grove, C. Hamadache, D. H. Hartmann, M. Hayashida, J. Isern, G. Kanbach, J. Kiener, J. Knödlseder, C. Labanti, P. Laurent, O. Limousin, 
F. Longo, K. Mannheim, M. Marisaldi, M. Martinez, M. N. Mazziotta, J. McEnery, S. Mereghetti, G. Minervini, A. Moiseev, A. Morselli, K. Nakazawa, P. Orleanski, J. M. Paredes, B. Patricelli, J. Peyré, G. Piano, M. Pohl, H. Ramarijaona, R. Rando, I. Reichardt, M. Roncadelli, R. Silva, F. Tavecchio, D. J. Thompson, R. Turolla, A. Ulyanov, A. Vacchi, X. Wu, A. Zoglauer, The e-ASTROGAM mission. Exploring the extreme Universe with gamma rays in the $\mathrm{MeV}-\mathrm{GeV}$ range, Experimental Astronomy 44 (1) (2017) 25-82. arXiv:1611.02232, doi:10.1007/s10686-017-9533-6.

[2] N. Werner, J. Řípa, A. Pál, M. Ohno, N. Tarcai, K. Torigoe, K. Tanaka, N. Uchida, L. Mészáros, G. Galgóczi, Y. Fukazawa, T. Mizuno, H. Takahashi, K. Nakazawa, Z. Várhegyi, T. Enoto, H. Odaka, Y. Ichinohe, Z. Frei, L. Kiss, CAMELOT: Cubesats Applied for MEasuring and LOcalising Transients mission overview, in: Proceedings of the SPIE, Vol. 10699 of Society of Photo-Optical Instrumentation Engineers (SPIE) Conference Series, 2018, p. 106992P. arXiv:1806.03681, doi:10.1117/12.2313764.

[3] V. Schonfelder, A. Hirner, K. Schneider, A telescope for soft gamma ray astronomy., Nuclear Instruments and Methods 107 (1973) 385-394. doi: 10.1016/0029-554X(73)90257-7.

[4] R. W. Todd, J. M. Nightingale, D. B. Everett, A proposed $\gamma$ camera, Nature 251 (5471) (1974) 132-134. doi:10.1038/251132a0.

[5] A. C. Zoglauer, First light for the next generation of Compton and pair telescopes : Development of new techniques for the data analysis of combined Compton and pair telescopes and their application to the MEGA prototype, Ph.D. thesis, - (Jan 2006).

${ }_{630}$ [6] V. Tatischeff, et al., ComptonCAM, https://international. a andra.fr/sites/international/files/2019-08/Fiche\%20projet\% 20COMPTON-CAM\%20UK.pdf (2019).

[7] A. Gostojic, V. Tatischeff, J. Kiener, C. Hamadache, N. Karkour, D. Linget, 
X. Grave, L. Gibelin, B. Travers, S. Blin, Application of artificial neural network in 3D imaging with lanthanum bromide calorimeter, Nuclear Instruments and Methods in Physics Research A 787 (2015) 140-143. doi:10.1016/j.nima.2014.11.076

[8] A. Gostojić, V. Tatischeff, J. Kiener, C. Hamadache, J. Peyré, N. Karkour, D. Linget, L. Gibelin, X. Lafay, X. Grave, Characterization of $\mathrm{LaBr}_{3}$ :Ce and $\mathrm{CeBr}_{3}$ calorimeter modules for 3D imaging in gamma-ray astronomy, Nuclear Instruments and Methods in Physics Research A 832 (2016) 24-42. doi:10.1016/j.nima.2016.06.044.

[9] A. Ulyanov, O. Morris, O. J. Roberts, I. Tobin, L. Hanlon, S. McBreen, D. Murphy, N. Nelms, B. Shortt, Localisation of gamma-ray interaction points in thick monolithic $\mathrm{CeBr}_{3}$ and $\mathrm{LaBr}_{3}$ :Ce scintillators, $\mathrm{Nu}$ clear Instruments and Methods in Physics Research A 844 (2017) 81-89. arXiv:1701.05548, doi:10.1016/j.nima.2016.11.025.

[10] S. Delorme, R. Frei, C. Joseph, J. F. Loude, C. Morel, Use of a neural network to exploit light division in a triangular scintillating crystal, Nuclear Instruments and Methods in Physics Research A 373 (1) (1996) 111-118. doi:10.1016/0168-9002(95)01511-6.

[11] W. S. McCulloch, W. Pitts, A logical calculus of the ideas immanent in nervous activity, The bulletin of mathematical biophysics 5 (1943) 115133. doi:10.1007/BF02478259.

[12] N. M. Ball, R. J. Brunner, Data Mining and Machine Learning in Astronomy, International Journal of Modern Physics D 19 (7) (2010) 1049-1106. arXiv:0906.2173, doi:10.1142/S0218271810017160,

[13] F. Chollet, et al., Keras, https://keras.io (2015).

[14] The Theano Development Team, R. Al-Rfou, G. Alain, A. Almahairi, C. Angermueller, D. Bahdanau, N. Ballas, F. Bastien, J. Bayer, A. Belikov, A. Belopolsky, Y. Bengio, A. Bergeron, J. Bergstra, V. Bisson, J. Bleecher 
Snyder, N. Bouchard, N. Boulanger-Lewandowski, X. Bouthillier, A. de Brébisson, O. Breuleux, P.-L. Carrier, K. Cho, J. Chorowski, P. Christiano, T. Cooijmans, M.-A. Côté, M. Côté, A. Courville, Y. N. Dauphin, O. Delalleau, J. Demouth, G. Desjardins, S. Dieleman, L. Dinh, M. Ducoffe, V. Dumoulin, S. Ebrahimi Kahou, D. Erhan, Z. Fan, O. Firat, M. Germain, X. Glorot, I. Goodfellow, M. Graham, C. Gulcehre, P. Hamel, I. Harlouchet, J.-P. Heng, B. Hidasi, S. Honari, A. Jain, S. Jean, K. Jia, M. Korobov, V. Kulkarni, A. Lamb, P. Lamblin, E. Larsen, C. Laurent, S. Lee, S. Lefrancois, S. Lemieux, N. Léonard, Z. Lin, J. A. Livezey, C. Lorenz, J. Lowin, Q. Ma, P.-A. Manzagol, O. Mastropietro, R. T. McGibbon, R. Memisevic, B. van Merriënboer, V. Michalski, M. Mirza, A. Orlandi, C. Pal, R. Pascanu, M. Pezeshki, C. Raffel, D. Renshaw, M. Rocklin, A. Romero, M. Roth, P. Sadowski, J. Salvatier, F. Savard, J. Schlüter, J. Schulman, G. Schwartz, I. Vlad Serban, D. Serdyuk, S. Shabanian, É. Simon, S. Spieckermann, S. Ramana Subramanyam, J. Sygnowski, J. Tanguay, G. van Tulder, J. Turian, S. Urban, P. Vincent, F. Visin, H. de Vries, D. Warde-Farley, D. J. Webb, M. Willson, K. Xu, L. Xue, L. Yao, S. Zhang, Y. Zhang, Theano: A Python framework for fast computation of mathematical expressions, arXiv e-prints (2016) arXiv:1605.02688arXiv: 1605.02688,

[15] M. Berger, J. Hubbell, S. Seltzer, J. Chang, J. Coursey, R. Sukumar, D. Zucker, K. Olsen, Xcom: Photon cross section database (version 1.5), http://physics.nist.gov/xcom, [Online] Available: http://physics.nist.gov/xcom [2020, February 12] (2010). 